\title{
SIMULTANEOUS NEAR INFRARED SPECTROSCOPY AND ELECTROENCEPHALOGRAPHY: A PROSPECTIVE SCREENING TOOL FOR BRAIN TRAUMA/DISEASE
}

\author{
by \\ Joshua Lee \\ B.Sc., McMaster University, Hamilton, Canada, 2013
}

\author{
A thesis \\ presented to Ryerson University in \\ partial fulfillment of the \\ requirements for the degree of \\ Master of Science \\ in the program of \\ Biomedical Physics
}

Toronto, Ontario, Canada, 2017

(C) Joshua Lee 2017 


\section{Author's Declaration}

I hereby declare that I am the sole author of this thesis. This is a true copy of the thesis, including any required final revisions, as accepted by my examiners.

I authorize Ryerson University to lend this thesis to other institutions or individuals for the purpose of scholarly research.

I further authorize Ryerson University to reproduce this thesis by photocopying or by other means, in total or in part, at the request of the other institutions or individuals for the purpose of scholarly research.

I understand that my thesis may be made electronically available to the public.

\section{Joshua Lee}




\title{
Simultaneous Near Infrared Spectrometry and Electroencephalography: A Prospective Screening Tool for Brain Trauma/Disease
}

\author{
Joshua Lee
}

M.Sc., Biomedical Physics, Ryerson University, 2017

\begin{abstract}
This thesis reports the development of a novel screening tool for brain trauma and disease using a headset capable of taking simultaneous measurements of electroencephalography (EEG) and functional near infrared spectroscopy (fNIRS) with a focus on developing the EEG side of the headset. Procedures for removing artifacts were developed for both modalities. The headset's measurements were validated using a breath-holding task for fNIRS and an eyes open/eyes closed and trail making tasks for EEG. The eyes open/eyes closed $(n=7)$ and trail making tasks $(\mathrm{n}=11)$ were further analyzed as potential tasks for use in screening. Integrated alpha power of EEG signals were found to provide robust differences between the eyes open/eyes closed states of the brain. Alpha power was also found to provide robust differences between rest and early trail making states in the trail making task, whereas, high beta power did not for either task.
\end{abstract}




\section{Acknowledgements}

I would like to thank my supervisor, Dr. Vladislav Toronov, Associate Professor in the Biomedical Physics Department for his guidance and suggestions throughout this undertaking.

I would also like to express gratitude towards my supervisory committee, Dr. Yuan $\mathrm{Xu}$, Associate Professor in the Biomedical Physics Department, and Dr. Soosan Beheshti, Associate Professor in the Electrical and Computer Engineering Department. Special thanks to Dr. Beheshti for her help and critique in signal processing and data analysis.

This project would not have been possible without the help of my colleagues, Reyhaneh Nosrati, Ermias Woldemichael and Siwook Kim. 


\section{Table of Contents}

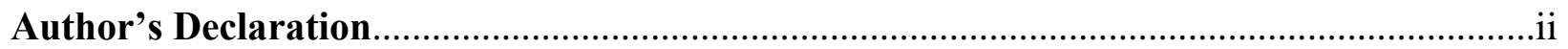

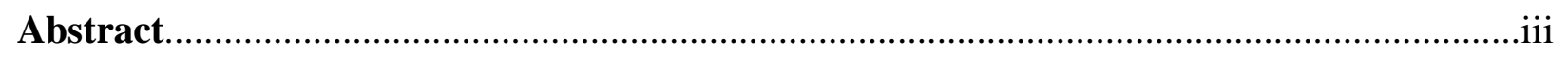

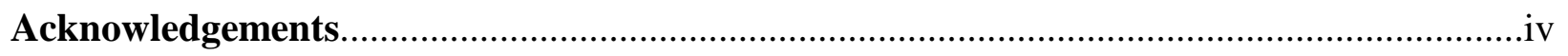

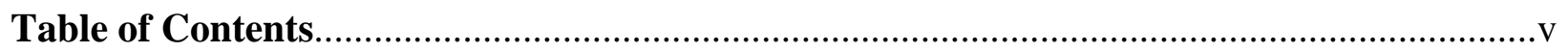

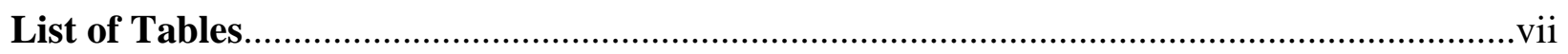

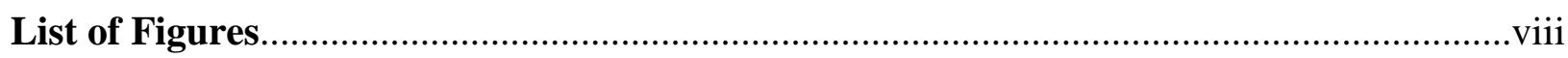

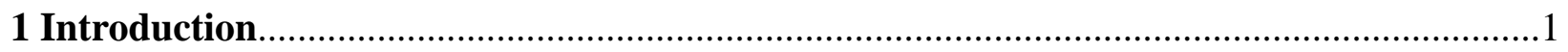

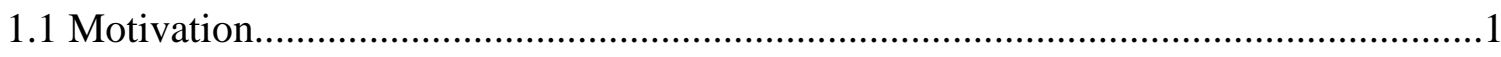

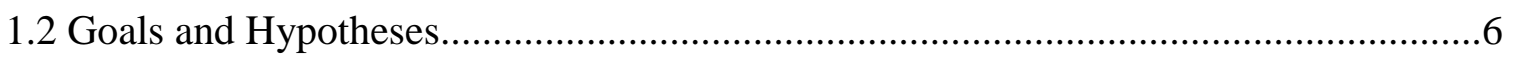

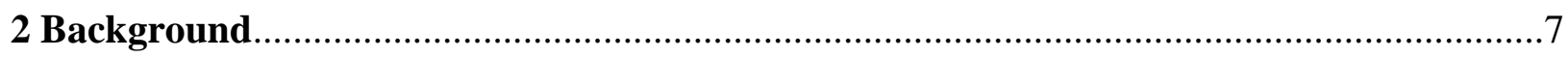

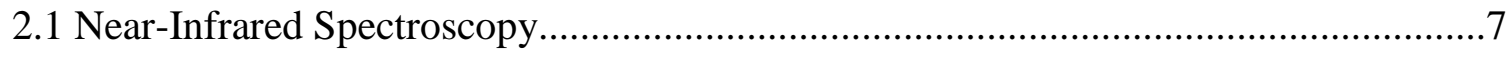

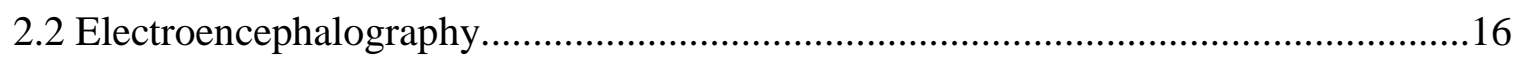

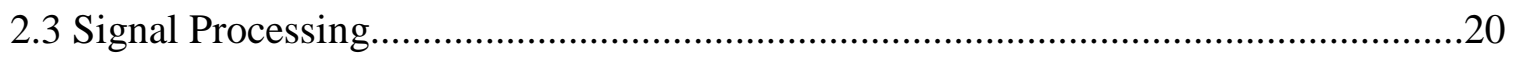

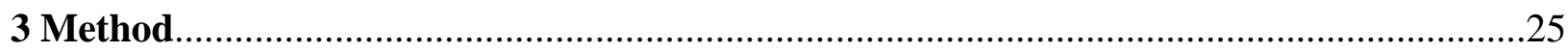

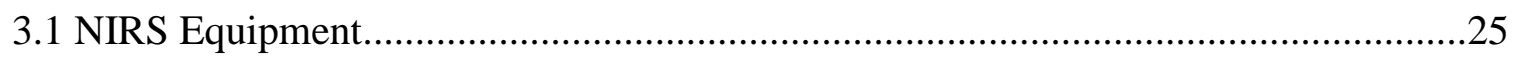

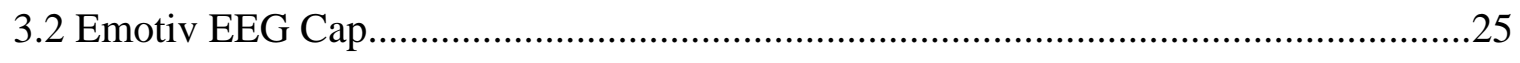

3.3 Combined NIRS and EEG Attachment..........................................................26 
3.4 Task Design.

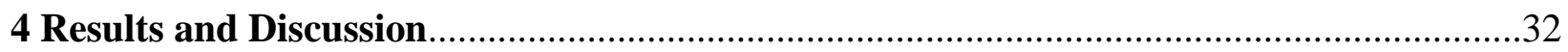

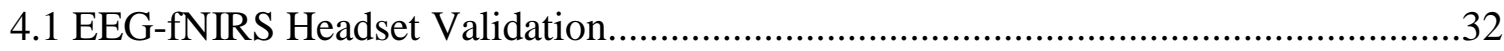

4.2 Developing the Eyes Open/Eyes Closed Task as a Prospective Screening Tool for

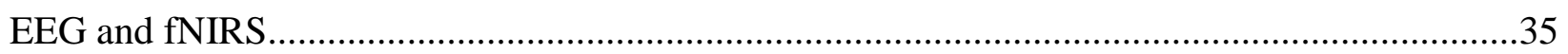

4.3 Testing EEG for Trail Making Task...............................................................42

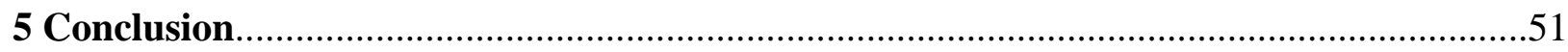

5.1 Summary of Results and Conclusion..............................................................51

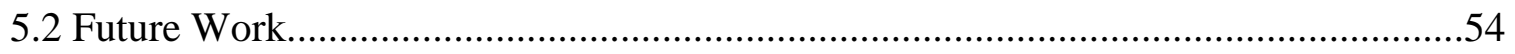

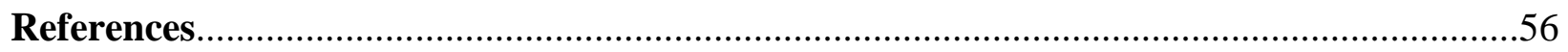




\section{List of Tables}

Table 4.1 Directionality of averaged powers from eyes open to eyes closed..............................38

Table 4.2 Calculated average difference between trails and rests before and after....................46 


\section{List of Figures}

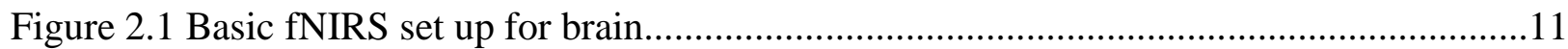

Figure 2.2 Diagram of the most probable pathway of light in biological tissue...........................12

Figure 2.3 Spectral data and fit fNIRS fitting algorithm.........................................................

Figure 2.4 Basic setup for EEG measurements...................................................................16

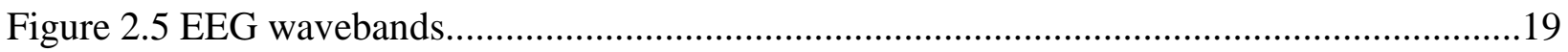

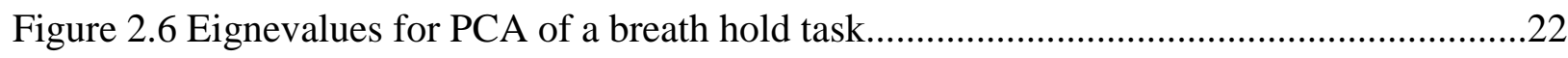

Figure 2.7 Removal of ocular artifacts in EEG signal using ICA...............................................24

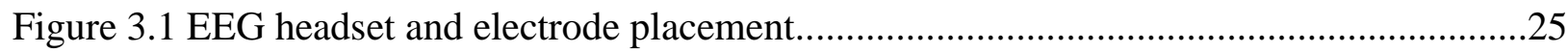

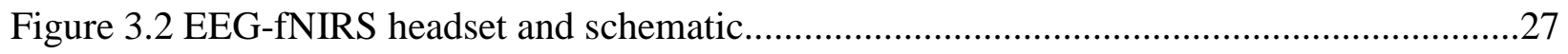

Figure 3.3 Trail making task example..................................................................................

Figure $4.1 \mathrm{HbO}_{2}$ and $\mathrm{HHb}$ concentration during breath hold task...............................................33

Figure 4.2 Alpha and high beta power during eyes open/eyes closed task....................................34

Figure 4.3 Averaged alpha and high beta powers during eyes open/ eyes closed task...................36

Figure 4.4 T-test results for individual channels for eyes open/eyes closed task...........................37

Figure 4.5 Averaged $\mathrm{HbO}_{2}$ and $\mathrm{HHb}$ concentrations during eyes open/eyes closed task..............39

Figure 4.6 Averaged power during trail making task................................................................43

Figure 4.7 T-test results for individual channels for averaged trails data......................................44 
Figure 4.8 T-test results for frontal trails vs rest data.......................................................47 


\section{Introduction}

\subsection{Motivation}

Between 2002-2007, there were 0.38 concussions per game in the National Football League. [1] A concussion, or mild traumatic brain injury (MTBI), is characterized by a disturbance in brain function usually by the violent jarring of the head, such as the result of a fall or in contact sports. [2] Common short-term effects of a concussion include a headache [3], dizziness, confusion, vision problems, lower cognitive function [4], and an irritable mood. Long-term symptoms include change in personality and sensitivity to light or noise.

Currently, the method of screening for a possible concussion in sports is the administration of a Sports Concussion Assessment Tool 2 (SCAT 2) test. [5] It is an assessment tool that checks for common post-concussion as well as an evaluation of motorphysiological responses as well as simple neurological test.

Should a doctor suspect a patient may have experienced a concussion after the physical and neurocognitive testing, images of the brain may be ordered. The two common modalities used for imaging the brain are computed tomography (CT) and magnetic resonance imaging (MRI). [6] A CT scan uses an x-ray beam to provide contrast using electron density. Many different angles are used to 'stitch' a three-dimensional image of the head. [7] For an MRI scan, the patient is placed in a strong magnet and the magnetic dipoles within the patient align with the strong field. The magnetic moments are then tipped using gradient coils and radiofrequency signal generated by the realignment of the magnetic moments is measured. Because MRI measures the relaxation of processing magnetic moments, only atoms with unpaired valence electrons can be measured, of which hydrogen dominates the signal. Thusly, the contrast in MRI is given by the proton density of the material. [8] 
Both modalities are incredibly effective at detecting major brain trauma, such as hemorrhaging in the brain. $[9,10]$ However, many concussions cause damage to the brain that is too small to detect using these modalities. When a CT scan and MRI images are taken, they often come back with negative results. However, a positron emission tomography (PET) scan reveals drastically reduced glucose uptake in a concussed brain, signifying disrupted brain activity after a concussion. [11]

It would then seem logical to use a PET scan for cases of concussion. However, like CT and MRI units, a PET scanner requires bulky equipment. An MRI machine requires a dedicated room shielded from outside electromagnetic radiation that may interfere with the radiofrequency signals and large cryogen tanks to cool the magnets. For PET and CT scans the unit must be in a shielded room to protect workers and patients outside of the room from the ionizing radiation produced by the scanner during operation.

In addition to requiring bulky equipment, PET, CT and MRI scans can be prohibitively expensive. In Canada, an average cost per scan is around $\$ 900$ for a brain scan at a clinic. [12] A CT scan in Canada can cost $\$ 895$ for a brain scan at a private clinic. [13] The average cost of a PET scan is $\$ 3000$ per scan. [14]

Despite having low success at diagnosing a concussion, CT and MRI scans are the two main modalities for screening concussions. The cost and low specificity of these scans make them less than ideal for diagnosing concussions or other mild changes in the brain's activity. Migrating to using PET scans would lead to better diagnosis of concussions, however the cost of scan and scarcity of scanners would not be able to serve the majority of people that are in need of a diagnosis; the scan would be either too expensive, too far away or the wait for an appointment would be too long. 
The goal of this work is to explore alternative methods and develop a preliminary screening tool for diagnosing concussions. The current methods, such as a neurocognitive test are subjective and a diagnosis could change between physicians administering the test. For example, the imPACT test was found to have a $47.5 \%$ chance of misdiagnosis. [60] CT and MRI scans seek to eliminate the subjectivity, but cannot discern between a concussed and healthy brain well. A PET scan detects reduced brain activity well, however centres that offer PET scans are scarce and are usually fully booked by oncology units at hospitals. The scans can also be prohibitively expensive for diagnosing mild trauma. An ideal candidate for a screening tool would be objective, without the need of interpretation by an individual; it would be able to measure a difference in brain activity between healthy and concussed patients; and it would be portable and inexpensive, able to be deployed at clinics or on-site at venues without the need of bulky equipment.

An alternative method currently being explored is functional magnetic resonance imaging (fMRI). [15] Unlike diagnostic MRI scans, an fMRI scan does not only reconstruct the structures of the brain, but measures quantities such as the blood oxygenation level dependent (BOLD) signal. The BOLD signal corresponds to brain activity through the uptake of blood in certain regions of the brain during different cognitive activities. [16] The main advantage of fMRI is that it detects changes in brain activity during these tasks, as well as the wealth of data already collected using MRI. However, the main drawbacks are the same as PET scans — the equipment is bulky, machines are not always available and the scans are expensive.

Two modalities that are of interest that satisfy the conditions of affordability, portability and objectivity are functional near infrared spectroscopy (fNIRS) and electroencephalography (EEG). 
NIRS measures the change in brain activity through the concentration of light absorbing molecules in the brain called chromophores by measuring the transmission of light through the brain in the near-infrared region of electromagnetic radiation. By measuring the concentrations as a function of time for chromophores such as hemoglobin and deoxyhemoglobin, brain activity can be inferred by the blood flow through the brain, much like fMRI. [17] Unlike fMRI, fNIRS does not require a room dedicated to it, there are no risks associated with a powerful magnet. NIRS, at a minimum, only needs an optical fibre leading from a light source and another optical fibre cable leading to a spectrometer that can bin light at various wavelengths, all of which has a much smaller monetary and physical footprint than the previously mentioned modalities.

EEG measures activity in neuron populations by measuring the electric potential differences at electrodes placed on the scalp. Unlike fMRI or fNIRS, the signal in EEG is not hemodynamic in nature, but of the activity of the neurons themselves, lending a different perspective in analyzing brain activity. [18] EEG also does not require bulky equipment with small caps available on the market for around $\$ 500$. [19]

In order to be used in a clinical setting, such as in a sports clinic which do not have access to CT or MRI facilities and where patients do not have an indefinite amount of time for screening, NIRS and EEG setups would need to be adapted to these environments. The number of channels for measurements would need to be reduced and setup times and procedures simplified. By measuring the chromophore concentrations using NIRS at a few locations on the head, such as on the forehead or occipital region, setup time would be reduced to a couple minutes. For EEG, setup time can also be reduced to a few minutes using a commercially available EEG cap. The main concern with reducing these modalities to just a few channels is that individually the data may not be sufficient. This is especially of concern with EEG; a higher density of electrodes 
can be used to average the signal over a region in the brain. With a low density of electrodes, the averaged signal is more susceptible to noise. Additionally, a lower density of electrodes also reduces the accuracy of localizing the source of phenomena detected using the EEG headset, though, that is beyond the scope of this project. [61] In fNIRS, the localization of the signal is in the most probable path of the light through the brain between the source fibre and the detection fibre. Additional fibres leading to detectors in the same region of the brain would decrease the noise in an averaged signal. Both modalities measure quantities related to brain activity, one through the cortical neuronal activity and the other through the hemodynamic response. This work seeks to determine if there is a comparable physiological change that can be measured in both modalities using a limited number of channels. By simultaneously measuring both NIRS and EEG on subjects, this work seeks to use the measurements in each modality to cross-validate the measurements in the other when used as a diagnosis tool.

Many different tasks have been designed for cognitive testing. For example, a task of drawing the time on the face of a clock is a neurocognitive task designed to screen for dementia. [20] However, the challenge for this work is to find a set of tasks that will give results in both NIRS and EEG as well as be a good candidate for screening concussions.

This work seeks to use both NIRS and EEG as a tool for screening for concussions. Before moving onto concussed patients, the two modalities must be tested together to determine if there is any physiological connection between them using healthy subjects. Various tasks will be given to subjects to determine if there is a correlation between the physiological changes seen in both modalities. Additionally, by using tasks designed to test cognitive function, the data can be cross-referenced with previous studies, such as fMRI studies, for similar tasks, leading to further validation of the data that was collected. 
This work is a first attempt at combining two simple modalities for use in screening of mild traumatic brain injuries where the standard protocols are currently difficult to administer in smaller clinics where the bulk of concussions are examined. By utilizing NIRS and EEG as a screening tool, this work seeks to improve the accessibility to objective-based diagnoses for concussions. In addition to using these two modalities as a screening tool for MTBIs, it expands upon the physiological connection between neuronal based activity and the hemodynamic response in the same region of the brain and further studies into other neurological diseases such as dementia may be possible.

\subsection{Goals and Hypotheses}

The goals of this work are to validate the measurements taken using the EEG-fNIRS headset as well as to test different protocols designed for cognitive studies and to adapt them to the headset. The ultimate goal of this work is to develop a cheap, portable alternative for screening mild traumatic brain injuries, possibly using some of the tasks tested herein.

My hypotheses are:

1) Simultaneous EEG and fNIRS using the fNIRS attachment for the Emotiv EPOC+ headset will produce meaningful results similar to previous studies in both modalities.

2) EEG and fNIRS data collected using the EEG-fNIRS headset will produce statistically significant differences between states of various tasks in healthy subjects. In order to test the eyes open/eyes closed task, the data should show a difference between the eyes open and eyes closed states in waveband powers and chromophore concentrations. Likewise, in a trail making task, the difference in each type of measurement should be significant between trails or between the trails and rest periods. 


\section{Background}

\subsection{Near Infrared Spectroscopy}

Near infrared spectroscopy refers to analyzing the output spectrum through a medium in the near-infrared region on electromagnetic radiation. In a biological setting, this window is between $700 \mathrm{~nm}$ and $1000 \mathrm{~nm}$. The dominant light-tissue interaction in the near infrared window is scattering, as such the light in tissue quickly becomes diffuse, increasing the pathlength through tissue and consequently absorption. At lower wavelengths, below $700 \mathrm{~nm}$, the absorption of light is dominated by hemoglobin and deoxyhemoglobin. At wavelengths greater than $1000 \mathrm{~nm}$, the absorption is dominated by water. Wavelengths between $700 \mathrm{~nm}$ and $1000 \mathrm{~nm}$ penetrate through tissue the deepest and are of the most interest, especially for applications in the brain where the light must penetrate through skin, skull, dura and finally brain. [21]

In the most basic setup of a NIRS experiment, a light source is connected to an optical fibre that leads to the head. This optical fibre feeds light into the head and into the brain. Another optical fibre is placed some distance away, in the case of the head this is around $30 \mathrm{~mm}$, [22] and the signal through the head and brain is sent to a spectrometer. The spectrometer bins the light according to its energy/wavelength and counts the number of photons it detects at a given wavelength over a specified amount of time called the integration time. This generates a spectrum of number of counts at a given wavelength over that course of time.

To understand how this spectrum corresponds to the concentration of certain chromophores, a good jumping point is the Beer-Lambert Law. Beer's Law is given by:

$$
I=I_{0} e^{-\alpha x}
$$


Where I is the intensity of the light, $\mathrm{I}_{0}$ is the incident intensity of the light before travelling through the medium, $\alpha$ is the molar extinction coefficient and $\mathrm{x}$ is the distance travelled through the medium.

Beer-Lambert's Law combines Beer's Law in Equation 1 with the definition of absorbance:

$$
A=\ln \left(\frac{I}{I_{0}}\right)
$$

Where $\mathrm{A}$ is the absorbance and $\mathrm{I}$ and $\mathrm{I}_{0}$ are the intensity of the light before and after the medium. Combined, Beer-Lambert's Law directly links the concentration of a chromophore to the absorbance of light through a medium containing that chromophore:

$$
\alpha=\frac{A}{x}=\varepsilon \boldsymbol{C}
$$

Where $\varepsilon$ is the extinction coefficient of the medium and $\mathrm{C}$ is the concentration of the absorber in the medium. [23]

It is important to note that the Beer-Lambert's Law does not apply to the situation of measuring light transmitted through the brain on the same side of the head. This is because BeerLambert's Law assumes that the light travels through the medium along an axis from one end of the medium to the other. The Beer-Lambert Law needs to be modified in order to be used for applications in the brain. [24] When using the Modified Beer-Lambert Law (MBLL), rather than relating the absolute optical density and absolute absorption, it relates the relative optical densities and relative absorptions. Unlike the traditional variant, the MBLL accounts for scattering within the medium by accounting for the mean pathlength. The MBLL must also be extended to a semi-infinite medium geometry. Though the MBLL is not used much in this work, it highlights the important relationship between the absorbance of the medium, which can be 
measured by the spectrometer via the relative intensities, and the concentration of the absorbers in the medium.

As previously stated, the molecules inside the medium that absorb or scatter the light are called chromophores. [25] There are various chromophores found within the human body. In the case of the brain, the main chromophores are hemoglobin and deoxyhemoglobin, Cytochrome-C oxidase and water. [26]

Hemoglobin is an important protein responsible for transporting oxygen and carbon dioxide through the body. The protein is a tetramer, consisting of 4 units. At the centre of each unit a heme group with an iron core accepts an oxygen molecule. [27] Deoxyhemoglobin is the variant of the protein that does not have any oxygen bound. When oxygen binds to an iron core within a heme group, the protein undergoes a conformational change that increases the association of the protein to additional oxygen molecules, with each oxygen added increasing the association of oxygen to the hemoglobin protein. [28] This conformational change between deoxyhemoglobin and oxyhemoglobin is responsible for the differences in the absorption spectra between the two different species of hemoglobin. An oxyhemoglobin concentrations increase and deoxyhemoglobin concentrations decrease indicates an increase in blood oxygen saturation. [29]

Another chromophore that is important to account for in the brain is Cytochrome-C oxidase. Cytochrome-C oxidase is a protein found within the membrane of mitochondria and contributes to the electron transport chain for the production of ATP. [30] Cytochrome-C oxidase can be used as a marker for oxygen consumption at the cellular level. [31] Like hemoglobin, Cytochrome-C oxidase contains a heme group, however, instead of an iron core, a copper core is situated at the centre. [30] 
Each chromophore absorbs near infrared light differently. In a heterogeneous mix of chromophores, each chromophore will contribute differently to absorption of light at different wavelengths. In the case of one chromophore present, in an ideal case, only one wavelength of light needs to analyzed in order to determine the concentration of a chromophore. Using one wavelength will not give enough data to determine the concentration if there is a mix of chromophores; this is especially important because of the two common species of hemoglobin in blood.

In a multispectral approach, such as in a Nonin pulse oximeter, instead of using one wavelength, multiple wavelengths are used to determine the concentrations of multiple chromophores. In the case of a pulse oximeter, the chromophores being measured would of oxyhemoglobin and deoxyhemoglobin. [32] It is assumed that the absorbances of each chromophore add linearly. Therefore, for two chromophores, at least two different wavelengths are needed to determine the concentration of each chromophore present. This method can be applied in an ideal case where the differences in the absorptions are large and the signal is strong.

However, for applications of NIRS in the brain, this method will not work. The signal must travel through the skull and brain and back. It is also further complicated by the fact that the concentration of Cytochrome-C oxidase is also very low. The primary area of concern is that the signal to noise ratio is low in these cases and taking measurements at one wavelength for each chromophore will give rise to very noisy results. In order to alleviate these issues, a hyperspectral approach can be taken.

In a hyperspectral approach to NIRS, or any waveband of light, rather than using discrete wavelengths to determine the concentrations of different chromophores present in a sample, an entire waveband of light is taken as a 'fingerprint.' In the case of the near infrared region, the 
wavelengths between 700 and $1000 \mathrm{~nm}$ are used. The advantage of using hyperspectral nearinfrared spectroscopy (hNIRS) over a multispectral approach is that is it less susceptible to the effects of poor signal-to-noise as it uses a broad range of wavelengths to fit the concentrations of the chromophores over rather than calculate the concentrations using Beer-Lambert's Law. [33] In a simple NIRS setup, a fibre optic cable feeds light into the medium and a fibre optic cable then collects light sends it to a spectrometer. In the case of setting up a NIRS experiment to measure chromophore concentrations in the brain, extra considerations are needed.

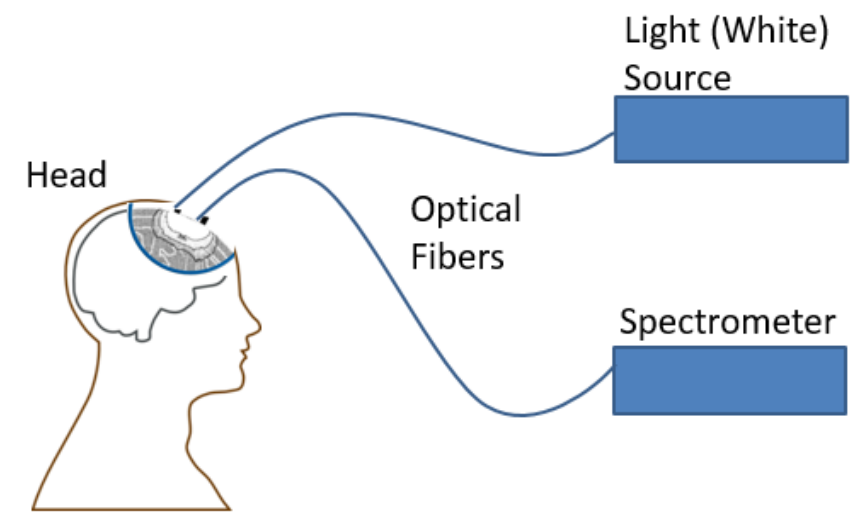

Figure 2.1. Basic fNIRS set up for brain.

First, because the fibre optic cables are placed on the same side of the head, the distance between the two cables is not the shortest distance between the two cables; that is to say, the most probable path will not be along the surface of the scalp. As light enters the head, it will either scatter or be absorbed by the medium. In biological media, there tends to be forward scattering, however after many scattering events, the overall effect is that the light can be said to be scattered isotropically - as if the scattering had no preferential direction. [34] NIRS is said to be a diffuse optical technique [35] and as such, this condition must be met, that is to say, the pathlength between the two optical fibres must at least the mean free path, or $1 / \mu_{\mathrm{s}}{ }^{\prime}$, where $\mu_{\mathrm{s}}{ }^{\prime}$ is the reduced scattering coefficient. [36] 


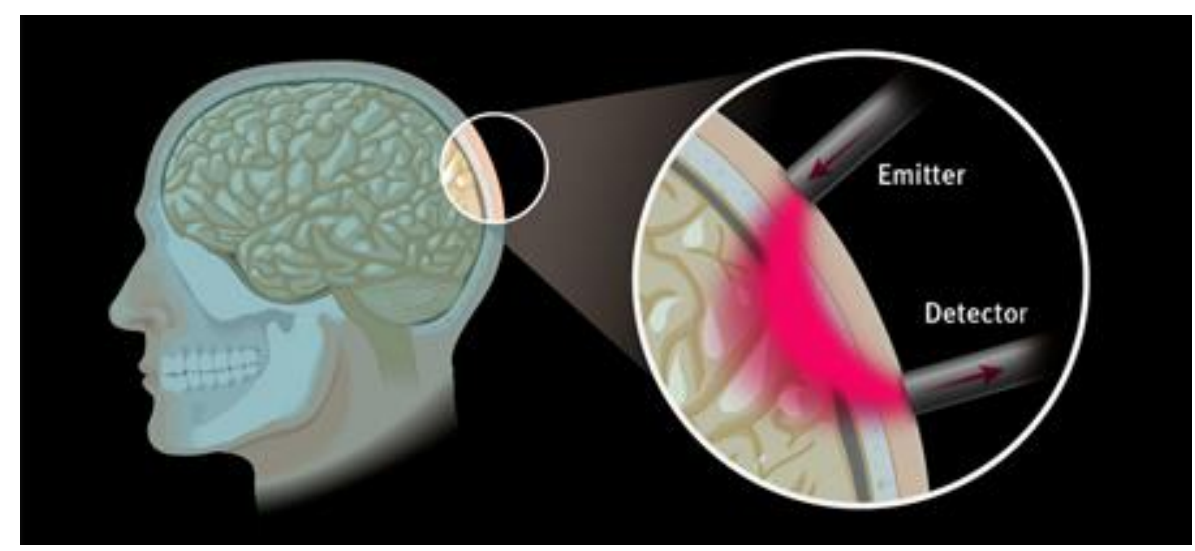

Figure 2.2. The most probable path light travels between the source and detector fibres in the head follows a 'banana' curve. [37]

But, what path does the light take to go from the source fibre to the detector? In truth, the light can take any path, be it from one end of the head to the other and back, or even a straight line between the source and detector fibres. However, due to the nature of the scattering events in the medium, the most probable path from one detector to the other is in a path similar in shape to a banana. [22] Because of this shape, the fibres leading to the source and the detector must be sufficiently far apart such that the most probable path will penetrate deep enough into the head to go through the brain, else the photons detected by the detector will most likely be the signal from the skin, skull and dura rather than the brain which is the intended target. It is also important to note that the head is not an 'infinite' medium. That is to say, that light that reaches the surface of the head will not scatter back into the head. By accounting for both the semi-infinite medium condition and the shape of the most probably pathlength, the diffusion approximation equation for a semi-infinite medium is given by:

$$
()=\frac{2}{(4)^{2}} \frac{S}{D} \frac{\exp \frac{a}{D} \div}{3} 1+\frac{a}{D} \div\left(z_{0}+z_{b}\right) \quad z_{b}+3 D 1 \frac{\left(z_{0}+z_{b}\right)^{2}+3 z_{b}^{2}}{2^{2}} 3+\frac{2 \frac{a}{D}}{1+\frac{a}{D} \div}
$$


Where $\phi$ is the fluence, $\rho$ is the distance between the source and detector fibres, $\mu_{\mathrm{s}}$ ' is the reduced scattering coefficient given by $\mu_{s}{ }^{\prime}=(1-g) \mu_{s}$, where $\mu_{\mathrm{s}}$ is the scattering coefficient and $\mathrm{g}$ is the anisotropy coefficient, $\mathrm{D}$ is the diffusion coefficient given by $D=\frac{1}{3\left(\mu_{a}+\mu_{s}^{\prime}\right)}$, where $\mu_{\mathrm{a}}$ is the absorption coefficient, $\mathrm{S}$ is the source strength, $\mathrm{Z}_{0}$ is the depth at which all photons are considered to be scattered given by $Z_{0}=3 D$ and $Z_{\mathrm{b}}$ is the distance between the extrapolated boundary and the physical surface given by $Z_{b}=2 D n_{\text {rel }}$ where $\mathrm{n}_{\text {rel }}$ is the relative index of refraction. [38] The value of $Z_{0}$ and $Z_{b}$ harkens back to the mathematics involved in deriving the diffusion equation where a pencil beam at the surface of a semi-infinite medium can be approximated by two point sources in an infinite medium using the mirror method (a first-order Taylor expansion). The extrapolated boundary refers to the boundary that is considered to have no fluence and the physical boundary refers to the surface of the semi-infinite medium. [39] When taking measurements, as the blood oxygenation or different areas of the brain are activated, it is assumed that the concentrations of different chromophores will change. More Cytochrome-C oxidase would indicate mitochondrial activity in the region the light passes through. Increased oxyhemoglobin and decreased deoxyhemoglobin would indicate an increase in blood oxygen saturation. As these processes occur, the signal that reaches the spectrometer will change. Rather than use an absolute approach in which the source strength is needed, a differential approach to NIRS can be taken. Using Equation 3, the differential version for the absorption coefficient becomes:

$$
\Delta \mu_{a}(t)=\Delta\left[\mathrm{HbO}_{2}\right]_{t} \varepsilon(\lambda)_{\mathrm{HbO} 2}+\Delta[\mathrm{HHb}]_{t} \varepsilon(\lambda)_{H H b}+\Delta[o x-C C O]_{t} \varepsilon(\lambda)_{C C O}
$$

Where the three chromophores present are oxyhemoglobin $\left(\mathrm{HbO}_{2}\right)$, deoxyhemoglobin $(\mathrm{HHb})$ and the oxidised variant of Cytochrome-C oxidase (ox-CCO). [38] 
To find the magnitude of the change in the absorption coefficient, between two time points in the dataset that is collected of an entire NIRS spectrum, the data at the two time points is divided and the $\log$ is taken of the quotient. This is then compared to the log of the quotient of the expected spectra at each time point and then these expected values are fit to match the data.
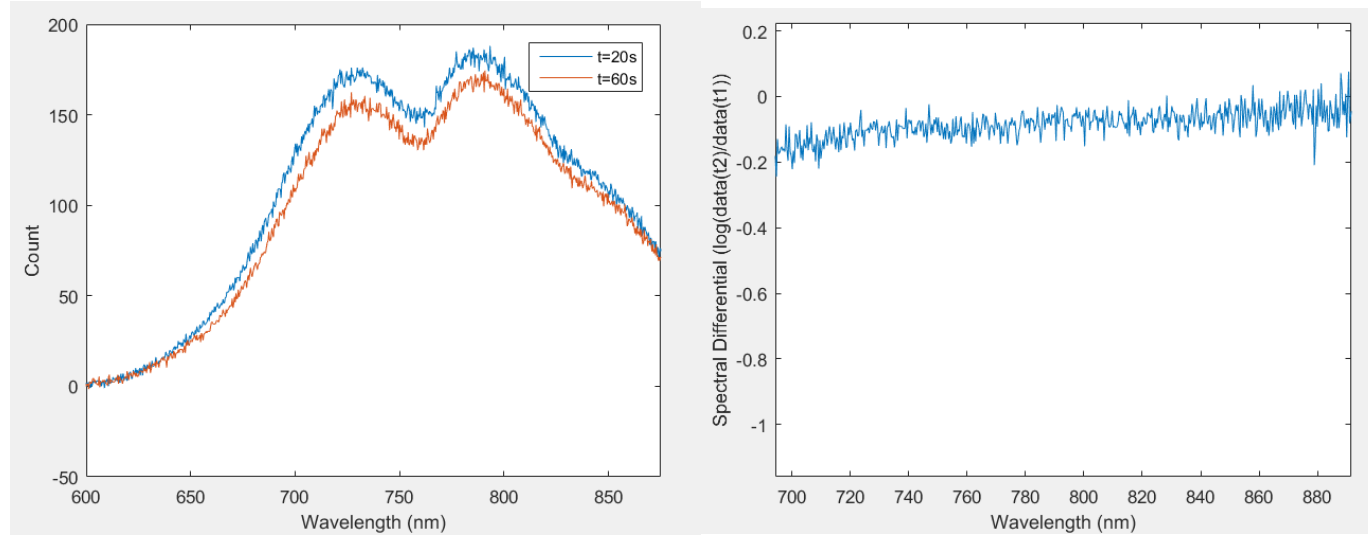

$$
\ln \frac{\psi_{\lambda}\left(\mu_{a}+\Delta \mu_{a}, \mu_{s}^{\prime}\right)_{t}}{\psi_{\lambda}\left(\mu_{a}, \mu_{s}^{\prime}\right)_{t_{0}}} \longrightarrow \ln \left[\frac{\operatorname{Data}(\lambda, t)}{\operatorname{Data}\left(\lambda, t_{0}\right)}\right]
$$

Figure 2.3. Left: Spectral data of intensity a function of wavelength during a breath-hold task. Shown are spectra during a breath-hold ( $\mathrm{t}=20$

s) and during rest $(t=60 \mathrm{~s})$. Right: The spectral differential of the two curves; the $\log$ of the spectrum at $\mathrm{t}=60 \mathrm{~s}$ divided by the spectrum at $\mathrm{t}=$ 20 s. Bottom: Calculated data is compared to collected data and parameters are changed to match the collected data.

Using Equation 5 and the known absorption coefficients of $\mathrm{HbO} 2, \mathrm{HHb}$ and $\mathrm{CCO}$, the values of $\Delta\left[\mathrm{HbO}_{2}\right]_{\mathrm{t}}, \Delta[\mathrm{HHb}]_{\mathrm{t}}$ and $\Delta[\mathrm{ox}-\mathrm{CCO}]_{\mathrm{t}}$ can be estimated to fit the data. A least-squared fit can be used to minimize the distance between the spectral differentials generated by the estimation of the concentration of the chromophores and the measured data. 
The resulting output is a set of concentrations of chromophores as a function of time. When compared to certain stimuli, a change in the concentrations of the chromophores can reflect the physiological changes happening in the analyzed tissue. It is the goal of this work to attempt to tie the results of the hemodynamic response ( $\mathrm{HbO} 2$ and $\mathrm{HHb}$ concentrations) to the neuronal response measured using electroencephalography.

The main advantage of using fNIRS is that it is less susceptible to artifacts due to environmental reasons that EEG, such as movement of the head or tongue. Another advantage of using fNIRS over EEG is that the signal is localized to the path between the source and detector fibres, rather than the delocalized electrical signals measured at the scalp using EEG. However, due to the constraints of taking NIRS measurements on the brain, the temporal resolution is poor when attempting to obtain a good signal, the spatial resolution is poor due to the depth needed for the light to penetrate into the brain, and NIRS does not measure brain activity directly, rather it measures the hemodynamics of the brain. 


\subsection{Electroencephalography}

Electroencephalography (EEG) refers to the measurement of bulk electrical potential differences at the surface of the scalp. [18] Several electrodes are placed on the scalp and measurements are taken simultaneously. In the most basic setup, only two electrodes are needed. One electrode is placed anywhere on the head and serves as a reference point. The other is placed at a different location on the head and the voltage difference is measured. In practice, many electrodes are placed at precise locations situated in different regions of the head. The largest regions are the frontal, temporal, parietal and occipital regions. With more electrodes, regions in between the major regions can also be examined, such as the fronto-temporal region.

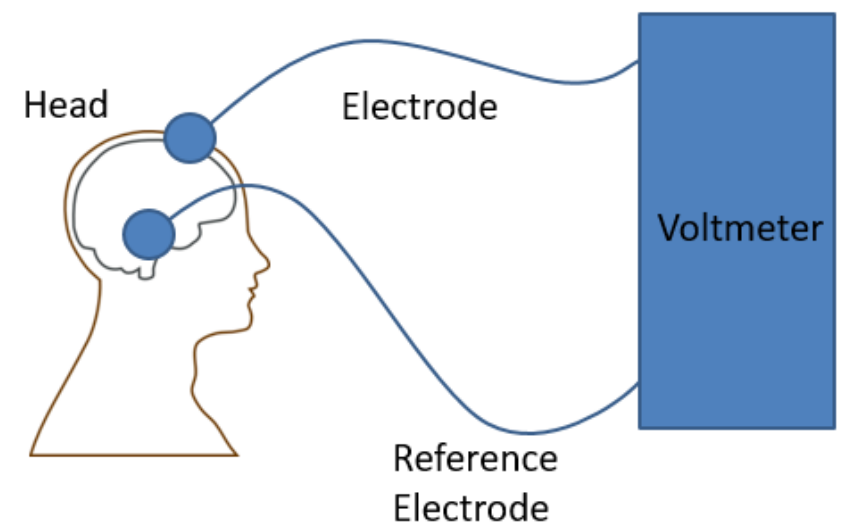

Figure 2.4. Basic setup for EEG measurements.

The very basis of EEG measurement lies in the electrical potential generated by neurons as they fire and conduct signals in the brain. [40] A neuron is special type of cell found in the nervous system; the central and peripheral nervous systems both contain neurons. A typical neuron consists of the soma, which houses the nucleus and is the main of the cell, dendrites which receive signals from other neurons. The axon transmits the signal towards the axon terminal. The axon terminal of one neuron and the dendrite of another neuron meet at a junction called a synapse, where signals are transmitted. It is the method in which a signal is conducted 
along an axon that is responsible for the electrical signal measured on an electrode during EEG measurements. An electrical potential travels along an axon by creating a potential gradient across the cellular membrane in the axon. When the signal is conducted along a stretch of the axon, ion channels are activated once a certain threshold is reached. Once the threshold has been reached, the pumps bring positively charged sodium ions into the cell, reversing the polarity of the gradient across the cellular membrane. As the ions diffuse inside the membrane, they cause adjacent rejoins to also reach the threshold potential and the signal is thus conducted. [41] When taking measurements of electrical potential, the bulk flow of ions into and out of the cellular membrane are ultimately responsible for the signal. [40]

Due to the thickness of the skull, neurons, even those in the cortex of the brain, are too far away from the electrodes to produce a strong signal. Rather than measure a single neuron firing, which would require the electrode to be extremely close, even in contact, with the neuron, measurements at the scalp measure the signal due to bulk conduction. As multiple neurons fire at the same time, the movement of ions outside of the cells causes the ions in the nearby vicinity to also move along the gradient. This bulk movement of ions is then what is measured by the electrodes. [40] The organization of neurons is also critical for this phenomenon. If all the neurons are not working synchronously, the signal will be minimal. [18] The brain is organized into two types of matter, grey matter which lies in the surface, or cortex, of the brain and white matter which lies in the interior of the brain. The grey matter in the brain contains the dendrites, soma and axon terminals of the neurons while the white matter on the interior of the brain contains axons in which the signal is conducted across different regions of the brain. [41] Because of this organization, in which dendrites lie in the cortex and axons in the interior, if multiple neurons fire at the same time in the same vicinity, then the resulting change in the local 
electrical potential is strong enough to be detected at the surface of the scalp by the electrodes. A type of neuron that satisfies this condition are neurons called pyramidal neurons. Pyramidal neurons are found in hippocampus, amygdala and most importantly in the cerebral cortex. [42]

The electrical signal is picked up by electrodes that are situated at the scalp. In some EEG caps, there are hundreds of electrodes. Each electrode measures the local scalp voltage generated by the brain underneath, but can also pick up other signals such as motion in the eyes and tongue as well as electromagnetic interference from the $\mathrm{AC}$ current running through power lines. [43] These sources of noise will be discussed later.

All EEG measurements are done so with respect to a reference point. The reference point can be any single electrode placed anywhere on the head. The reference is sometimes also taken as an average over several electrodes and can even be taken to be the average over all electrodes. In a typical application, EEG measurements are made in reference to an electrode close to the ear in the temporal region. [40]

Each channel on an EEG headset or cap measures the electrical potential deflection with respect to the reference channel. [18] If the local potential underneath an electrode increases with respect to the reference channel, the voltage reading for that channel will deflect upwards. Similarly, if the electrical potential underneath the electrode decreases with respect to the reference channel the signal will deflect downwards. It is important to note that a flat line in a signal may not necessarily mean that there is no activity underneath the electrode, it may mean that the electrical potential is the same as the reference.

The deflections seen on EEG readings typically happen periodically. It is thought that for small neural networks, the oscillations are fast, and when the oscillations are slow, the network is large. These oscillations arise from neurons synchronizing. [62] For very fast oscillations such as 
gamma waves, between $25-100 \mathrm{~Hz}$, typically around $40 \mathrm{~Hz}$, the oscillations have been found to be present during meditation. Interestingly, it has been shown that during sleep, gamma waves have been present during cases of lucid dreaming. Investigators then stimulated the brain during sleep with a $40 \mathrm{~Hz}$ wave. They found that the stimulation was able to induce lucid dreaming, highlighting the physiological importance of these oscillations. [46]

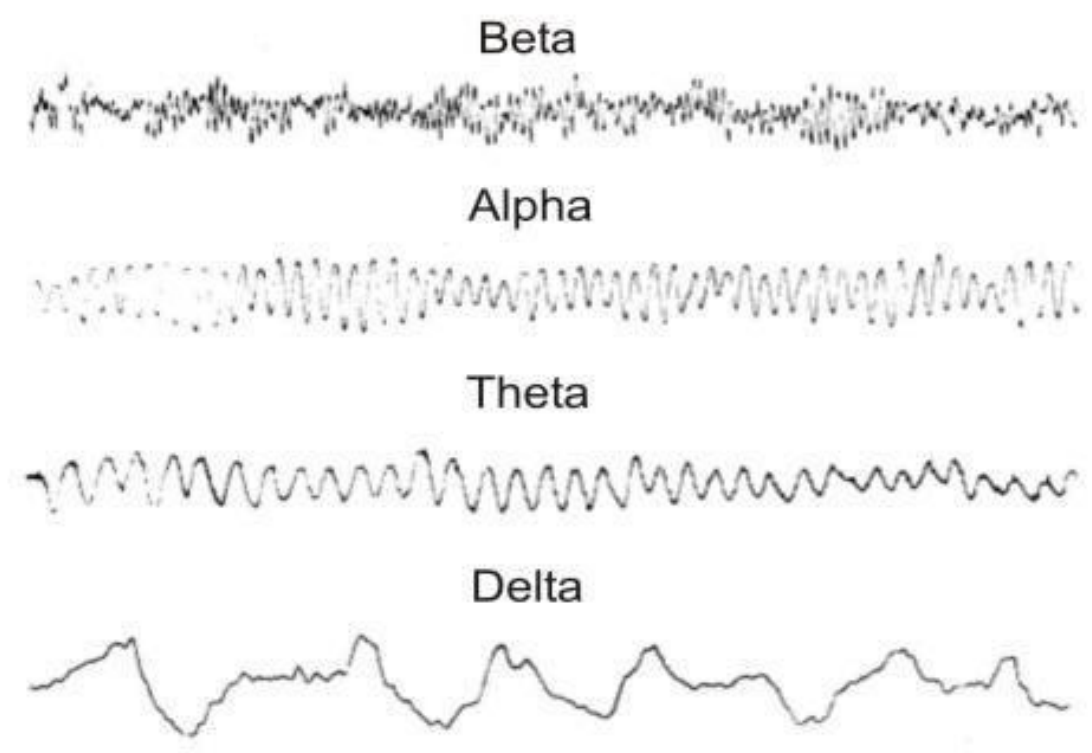

Figure 2.5. EEG wavebands. Alpha power is associated with a conscious eyes closed state. Beta power is associated with concentration and mental tasks.

Two wavebands that are of particular interest are alpha waves, between $7-13 \mathrm{~Hz}$ with a typical wave around $10 \mathrm{~Hz}$ and beta waves, between $12.5-30 \mathrm{~Hz}$. Alpha waves are associated with a conscious resting state, typically with the eyes closed and originate from the occipital region (the visual cortex). When the eyes are closed, the neural networks are able to synchronize due to lack of visual calculations being performed at the visual cortex. [62] Beta waves are associated with active concentration and vigilance. [44] 
To measure the power of each of the wavebands, the voltage can be transformed into the frequency domain using a Fourier transform to find the temporal frequencies of the output. Because the measurements taken are discrete, the Fast Fourier Transform is applied to the collected data. Spectral data can then be integrated over the wavebands to produce values for power. During different tasks, the power of each waveband may change, for example, during an eyes open and closed task, it is expected that the alpha power will increase when the eyes are closed when compared to when the eyes are open. By comparing the changes in the spectral power in the EEG signals and the concentration changes in fNIRS, a connection between the neuronal activity measured by EEG and the hemodynamic response detected by fNIRS can hopefully be established.

The main advantage of using EEG is that it has excellent temporal resolution and measurement are directly linked to the activity of the neurons. However, the signal in EEG is susceptible to noise due to the environment such as eye and muscle movements as well as poor spatial localization of the signal. [43]

\subsection{Signal Processing}

Noise is present in any measurement taken and steps are needed in order to reduce the effects on the signal in order to extract meaningful conclusions from the data.

In hNIRS, a major source of noise stems from the equipment. In a spectrometer, even with no light incident on the detector, a small signal is still 'detected.' This is due to the dark current, a small current that runs through the device caused by the stochastic creation of electrons and holes in the depletion region of the diodes present in the device. [45] The dark current can be 
subtracted to reduce the noise introduced by the equipment, however, it does not completely eliminate it due to small fluctuations in the dark current as a function of time.

Another problem in NIRS concerning signal is the relatively low counts of photons due to the spacing between the source and detector fibres. Because the light diffuses inside the tissue of the head, very little of the original light from the source reaches the detector. A photon reaching the detector from the source is considered to be a random event, independent of other photons. As such, the noise from collecting light follows a Poisson distribution with a standard deviation of the photon count equal to the square root of the count. In order to reduce the effect of this noise, many counts are needed. The integration time, the amount of time used to collect light at the detector, can be increased to achieve this. This successfully reduces the effects of noise in the signal, however it reduces the temporal resolution of the output.

Even after increasing the integration time, the signal may still have noise in it. First, the data can smoothed spectrally. Smoothing takes a weighted average around each data point, reducing the effects of noise. Sometimes the signal may also drift over time. Polynomial detrending can remove the drift by subtracting a fitted polynomial to the data to analyze the fluctuations around the trend. [47]

In addition to smoothing the data spectrally and detrending the data temporally, an independent component analysis can be performed on the data. Independent component analysis (ICA) is a method of separating a signal into many components. The classical problem that can be solved using ICA is the cocktail party problem. A room is filled with microphones and an equal amount of people speaking. Each microphone individually will only be able to pick up each voice as a mix, with each microphone detecting different levels of volume of each voice depending on proximity. Using ICA, each voice can be sorted out individually, with each 
microphone contributing to each component. [48] Another method for separating data is principle component analysis (PCA). Both methods create orthogonal sets of data, but ICA differs from PCA in that it seeks to minimize the amount of mutual information held within each component. On the other hand, a PCA algorithm seeks to maximize the amount of information that is in each component that has not been calculated. [49] In the case of a cocktail party, ICA's first component would be the loudest voice whereas PCA's first component would be the average sound at the party.

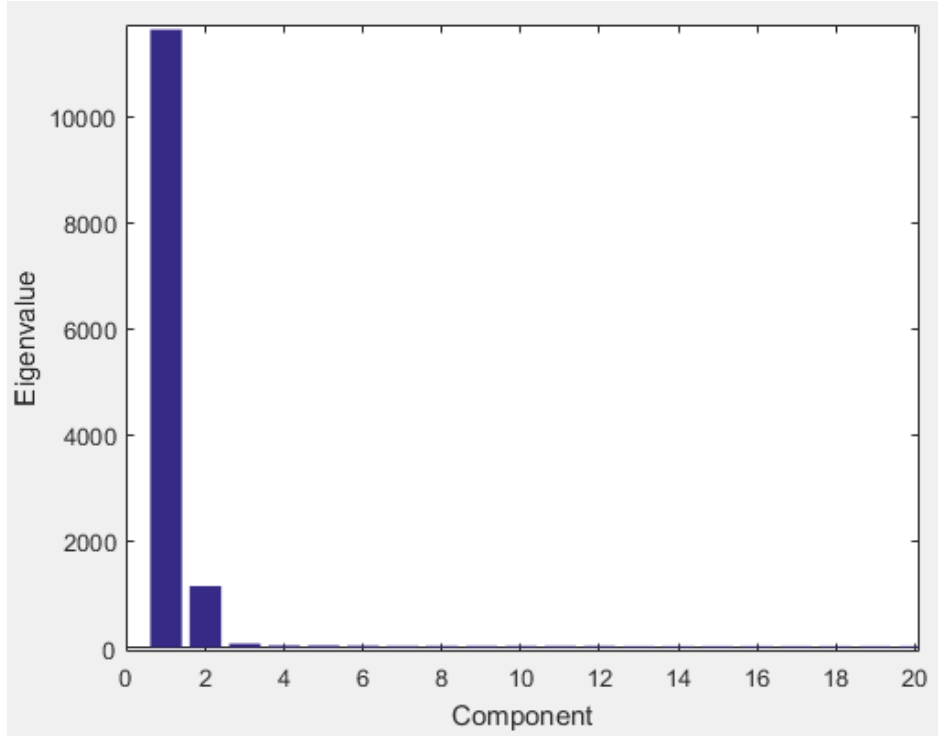

Figure 2.6. First 20 eigenvalues of PCA performed on a breath-hold task. Demonstrates that most of the data is contained within the first few components and that dimensionality can be reduced before an ICA is performed.

In the case of NIRS data that may have noise, an ICA can be run on the data. Using the analogy of a cocktail party, each wavelength can be treated as a 'microphone' and counts as a function of a time at each wavelength is the mixed signal. However, this would create many different components, many of which hold little data. In this case, when there are many more components than needed, the dimensionality of the data can reduced. An effective way to retain 
most of the data is to first perform a PCA, which maximizes the amount of data in its early components. The first few components of a PCA can be taken while discarding the rest of the data and an ICA can then be run on the remaining components. This is mainly done to reduce the computation time needed as well as represent the data in only a few components without losing too much data.

In EEG, the signal is susceptible to outside sources, such as movement artifacts and electric signals that are generated by muscles and the eyes when they are activated. Because motion artifacts tend to be slow with respect to the oscillations typically seen in conscious EEG. To remove much of the noise introduced by motion artifacts, a high-pass filter can be applied. This has the drawback of also removing very slow oscillations that can be present in the signal, but these wavebands are typically more important during sleep rather than when a subject is awake.

Another source of noise in EEG is pickup of 50-60 Hz noise from AC power lines. The body acts as an antenna for line frequency noise. To remove this noise, a low-pass filter can be applied to remove high frequency noise.

However, even after applying both a high-pass and low-pass filter, there is usually still artifacts present in the output. To remove such artifacts, an ICA can also be applied to the readings off each channel. An ICA is run, treating each channel as a receiver and the electrical potential measured as function of time on each channel as the mixed signal. Components can then be analyzed to determine if they should be discarded. An obvious example of an artifact that is easily removed by ICA is the large electroocular signal introduced by the eye. [50] The component presents itself strongly in the frontal region of the brain and eye blinks can easily be seen in the signal as large peaks. While useful for cleaning the data, ICA can also be used for localizing the source of signals, though that is beyond the scope of this project. 


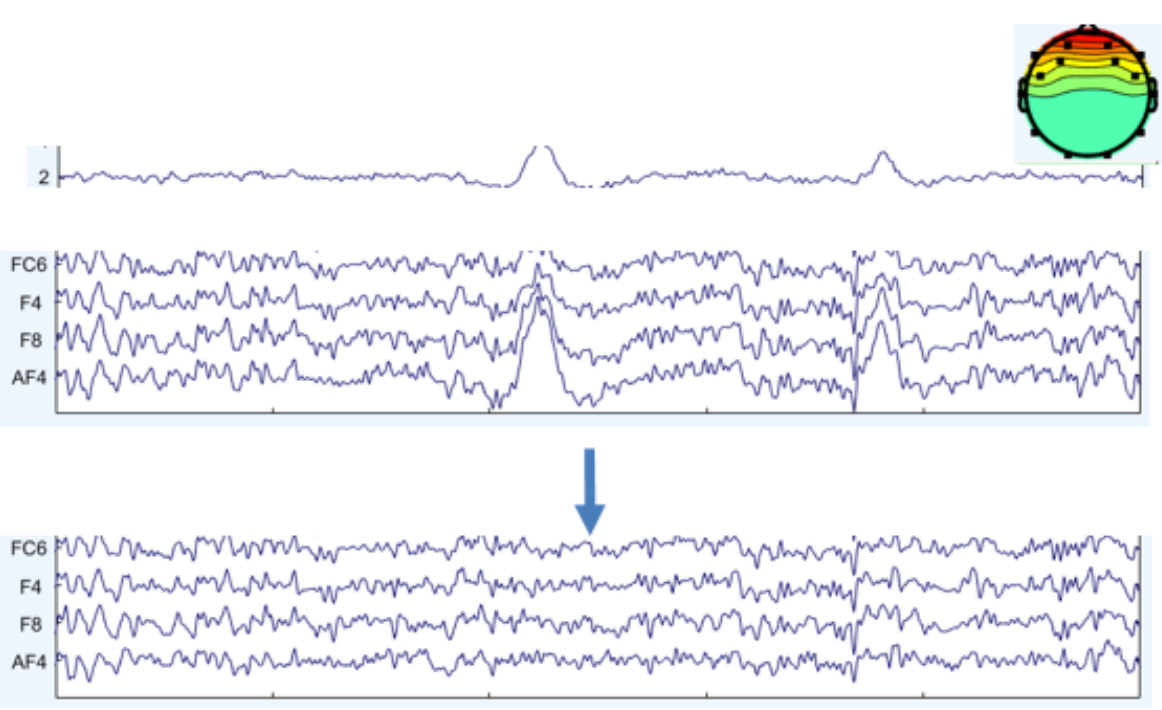

Figure 2.7. Removal of ocular artifacts in EEG signal using ICA.

After the signals have been 'cleaned,' they can then be processed as normal - fit using a diffusion approximation equation for NIRS to obtain chromophore concentrations, and Fast Fourier Transformed and integrated over wavebands for EEG to get spectral power. 


\section{Method}

\subsection{NIRS Equipment}

NIRS was performed using a white light source, Fiber-Lite DC-950 (Dolan-Jenner). 3 mm fibre optic cables (Dolan-Jenner) were used to feed light into the skull from the light source and another cable was used to collect the signal and send it to the spectrometer. The spectrometer used was a QE6500 (Ocean Optics) and a USB4000 (Ocean Optics). Each spectrometer was built specifically to measure in the NIR region. SpectraSuite (Ocean Optics) was used to record the data on computer.

\subsection{Emotiv EEG Cap}
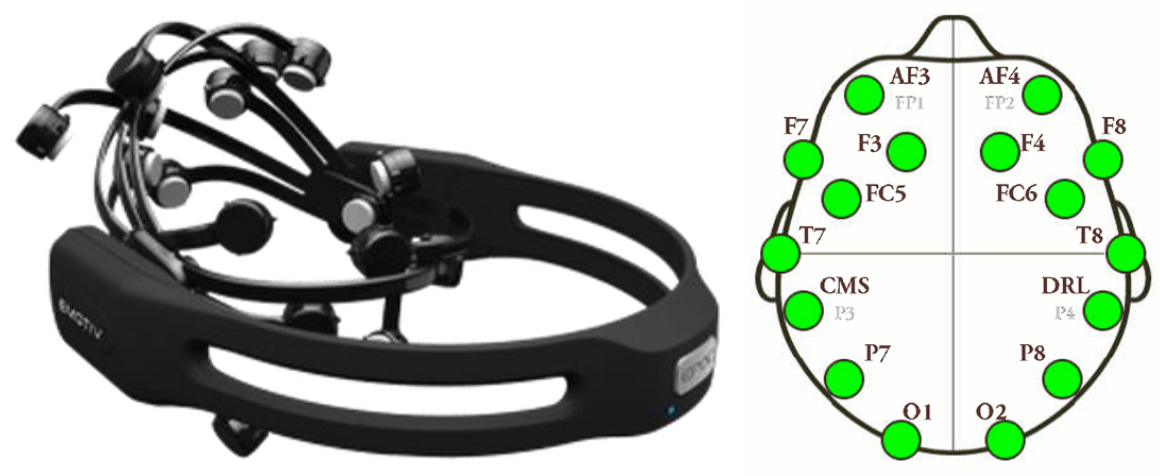

Figure 3.1. Left: Emotiv EPOC+ headset. Right: Electrode placement for EPOC+ headset.

EEG was measured using an Emotiv EPOC+ EEG headpiece. Unlike traditional EEG caps, the Emotiv EPOC+ does not use pads and gels to attach the electrodes to the head, rather the headpiece makes use of felt tips moistened with saline to lower the impedance of the electrodes. While saline does not conduct the electrical signal as well as a gel used for EEG, the time needed 
for setup is much less. The EPOC+ has 14 output channels and 2 reference channels. The output channels are situated at AF3, AF4, F7, F8, F3, F4, FC5, FC6, T7, T8, P7, P8, O1 and $\mathrm{O} 2$ around the periphery of the brain with no channels that lie on the crown of the head. The two reference channels are situated at $\mathrm{P} 3$ and $\mathrm{P} 4$ and are set up in a common-mode sense/driven right leg (CMS/DRL) configuration. This reference configuration, in addition to serving as the electrical potential difference each channel is compared to, is also designed in such a way that the circuitry can apply built in filters that can remove noise caused by $50 \mathrm{~Hz}$ and $60 \mathrm{~Hz}$ pick up from $\mathrm{AC}$ power sources. [19] Data was transferred to a computer via Bluetooth and collected using Test Bench (Emotiv).

\subsection{Combined NIRS and EEG Attachment}

An attachment to the Emotiv EPOC+ headpiece was commissioned and 3D printed to accommodate simultaneous collection of EEG and NIRS data. The attachments snapped onto the EEG headset at the F7 and F8 electrodes. Each attachment had two holes adjacent to the electrodes for the fibre optic cables to attach to the apparatus. The source to detector distance of the fibres was $3 \mathrm{~cm}$ to facilitate the optimal path through the head to get signal from the brain. The positions of the source and detector fibres positions were on either end of the electrode position, with the more lateral optode position further down than the medial optode. The source and detector fibres positions were chosen such that the most probably path that the light will travel through will be in the same position as the electrode. The frontal electrodes were chosen because of the lack of hair in the vicinity of the placement of the optodes. 
During set up, EEG positions were determined using an EEG cap to mark the positions of F7 and F8. These positions were vital to mark to ensure that the electrodes were in the same position each time. After marking, the Emotiv EPOC+ headset with the NIRS attachment was placed with the F7 and F8 electrodes aligned with the markers and the two rubber pads sitting on the mastoid process. The apparatus was held together using Velcro straps to fasten the headset, which must have the weight of the attachment compensated for, and to ensure a tight seal of the optodes.
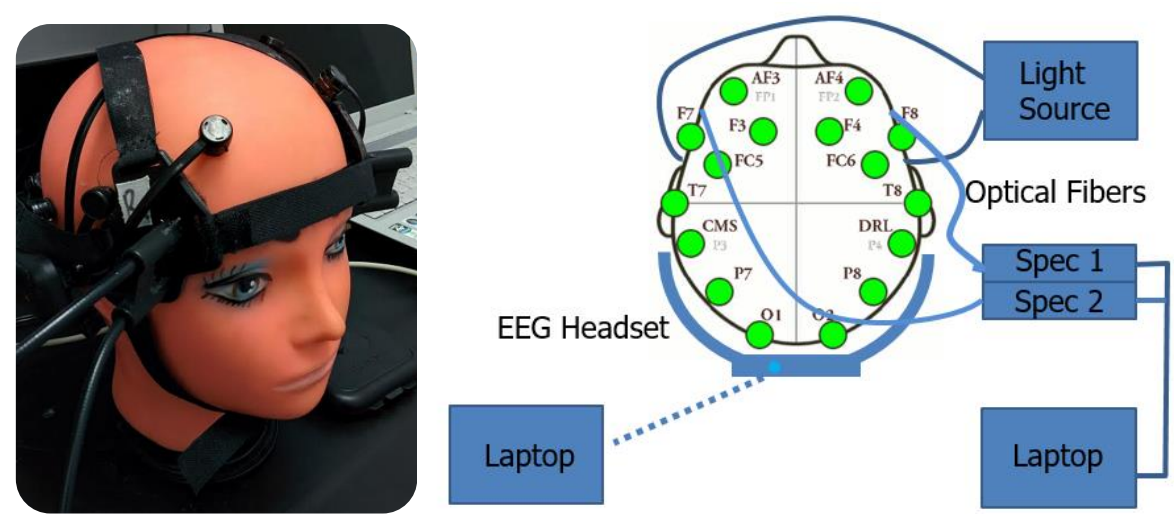

Figure 3.2 Left: EEG-fNIRS headset. Right: Schematic of lab setup for data collection.

The fibres were placed with the fibre leading to the light-source medial to the electrode and the fibre leading to the detector lateral to the electrode. NIRS signal was checked by verifying the maximum count above the baseline was at least 100. EEG signal was checked by ensuring that each channel's contact was adequate (positions will turn green on TestBench software.) 


\subsection{Task Design}

Various tasks were performed in order to test the physiological responses in both EEG and fNIRS.

Several tasks were intially implemented, however, some tasks were found to be inadequate for testing EEG and fNIRS signals. Several neurocognitive tasks designed for assessing concussions, provided by a concussion clinic, were adapted for use with the combined EEGNIRS headset. A memory recall task was implemented where subjects were tasked with recalling shapes; subjects were presented with a series of shapes and were asked to redraw the shapes some time later. A word recall task was also performed, with subjects presented with a list of words and then presented with a second list of words and asked if the word belonged to the first list or not. A number recall task was also performed; a list of numbers is given and the subject is asked to recite the list in reverse. All these tasks performed test a subject's memory as memory related problems are a hallmark of concussions. A reaction test was also performed, where subjects were to press different buttons depending on the colour of square presented.

Despite these tasks being often used to determine if the subject has a concussion through an assessment with a doctor or therapist, it was found that it did not translate well to the headset. The tasks were performed without breaks, meaning that other than placing markers in the data, there was little that could separate the data acquired through the different tasks. Additionally, there were frequent hand-offs in the process, with the tablet handed between the subject and the person administering the test. This lead to distortions in the data due to large artifacts caused by motion. Some tasks also required verbal feedback, this was disastrous for EEG because the signals in the channels would pick up the electrical signal generated in the tongue. These tasks 
were not selected for further testing due to lack of control for timings, movement of the body and no way to sort individual tasks as the tasks were performed consecutively without pause.

Rather than use a neurocognitive task developed for concussions in a clinic, the validity of the EEG and fNIRS signals can be determined by simpler tasks. For EEG, a simple eyes openeyes closed task can be implemented and for fNIRS, a breath hold exercise is performed. These tasks are physiological in nature rather than cognitive, but are suitable for testing the validity of the equipment's signals.

An eyes open-eyes closed task has the subject fixated on a visual cue, in this case a green light in the lab room in dim light, when the eyes are open. This ensures that the ocular artifacts introduced into the signal due to eye motion is minimized, as well as standardize the test to ensure that the data is comparable between subjects. The subject alternates between an eyes open state to an eyes closed state at set periods. This test is primarily tests the EEG signal. It is expected that the alpha power will increase during periods when the eyes are closed and decrease when the eyes are open, as alpha power corresponds to a rested, conscious state. This is due to the synchronization of the neurons in the visual cortex when the eyes are closed (no visual calculations). [62]

A breath hold test has the subject hold their breath for a short period of time at regular intervals. During the task, normal breathing is conducted by controlling the breath with a circle that the subject is fixated on. As the circle grows, the subject inhales and as the circle shrinks, the subject exhales. The subject then holds their breath for 16 seconds and the cycle repeats 6 times at 1 minute intervals. This task was designed at St. Michael's Hospital for fMRI patients and is displayed using E-Prime software. 

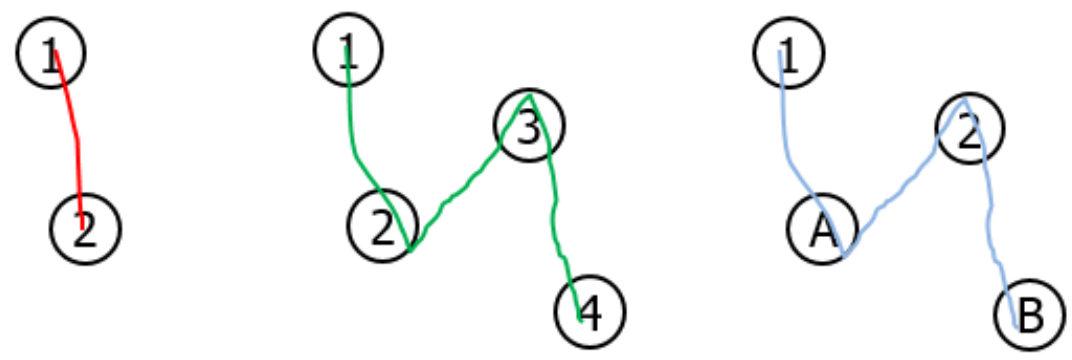

Figure 3.3. Examples of trails performed during a trails making task.

Left: Control task; a line was drawn between 1 to 2. Centre: Trails A task; the trail is drawn in increasing numerical order. Right: Trails B task; the trail is drawn in increasing alphanumerical order, alternating between numbers and letters.

Other E-Prime tasks that were also conducted were a clock drawing task, trail making task, and a letter cancellation task. A clock drawing task has the subject draw the face of an analog clock on a tablet, complete with all the numbers, and are tasked to draw in the time given. As a control, the subjects also trace a circle. This differentiates the signals received from the planning and concentration needed to draw the clock versus the simple task of drawing a circle. This task is primarily used in studies with dementia. [63] The letter cancellation task is designed to screen for deficits in a subject's visual scanning ability, typically seen in stroke patients. [64] During a letter cancellation task, a letter is designated to be struck out by the subject. An array of letters is presented to the subject and the subject then strikes out each letter in the array that matches the designated letter. As a control, the subject is presented with an array of letters and is instructed to strike through all the letters. 
For a trail making task, the subject is presented with three tasks in increasing difficulty starting with a control task of drawing a line between 2 bubbles. The second task requires the subject to draw a trail from the lowest numbered bubble to the greatest in sequential order and the third and most difficult task requires the subject to draw a trail between bubbles containing numbers and letters in increasing order, ex. 1-A-2-B-3-C etc. The trail making task primarily tests a subject's mental concentration and vigilance and have been used extensively for neuropsychological assessments. [65] The trail making task was chosen for further investigation because it reflects a wide variety of cognitive processes, something that a concussed patient would ideally show a vast difference in from healthy subjects. 


\section{Results and Discussion}

\subsection{EEG-fNIRS Headset Validation}

\subsubsection{Results}

In order to develop the EEG-fNIRS headset as a prospective screening tool for concussions, the measurements off of the headset first had to be validated to give expected results.

In the case of fNIRS, a breath hold task as described in Chapter 3 was used to determine if the signals collected from the headset with simultaneous collection of EEG and fNIRS data matched the expected behaviour from a breath hold task. Figure 4.1 shows the time course (6 minutes) of the calculated deoxy and oxyhemoglobin concentrations during the breath hold task. Signals were collected with a 500ms integration time to ensure that the signal through the head was large enough to minimize the effects of noise. Spectra were then smoothed spectrally (Matlab smooth function) and detrended using a polynomial detrend in Matlab to further reduce noise in the sample. An ICA (fastICA program suite in Matlab) was performed on the smoothed spectral data temporally. The data was first reduced to 3-5 dimensions in PCA (done through the fastICA algorithm) to reduce computation time and retain $95 \%$ of the data. Calculated components that contained artifacts, such as large jumps in the data due to initializing the dark current correction and motion artifacts. The remaining components were then back transformed to reproduce 'cleaned' spectra over the time course.

The cleaned data is then put through the fitting algorithm to determine the concentrations of oxyhemoglobin and deoxyhemoglobin. 


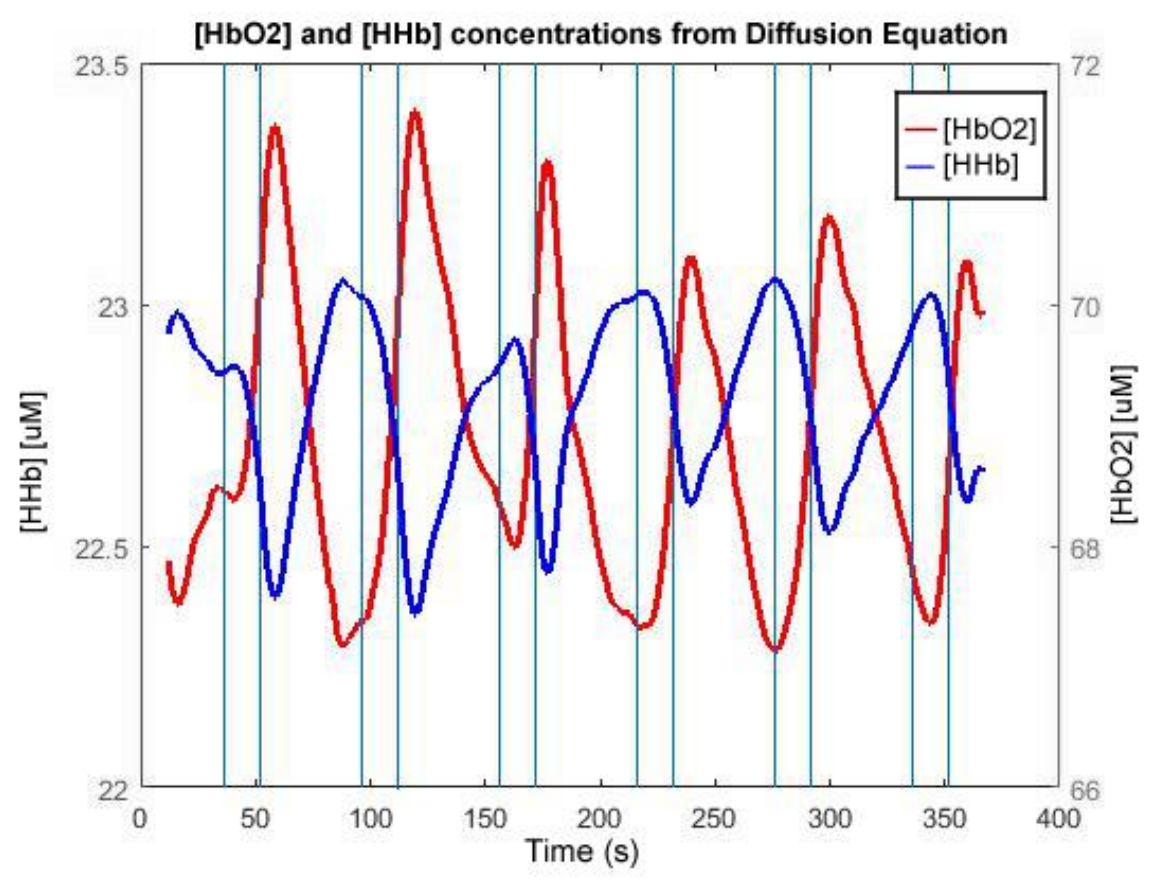

Figure 4.1. Oxyhemoglobin and deoxyhemoglobin concentrations as a function of time during a breath hold task. Task was moderated using EPrime software with 16 second breath holds highlighted.

For EEG, an eyes open and eyes closed task was developed to determine the robustness of the data collected. EEG data was collected simultaneously with fNIRS using the Emotiv EEGfNIRS headpiece. Scalp voltage was sampled at $128 \mathrm{~Hz}$ and collected for either $150 \mathrm{~s}$ or $600 \mathrm{~s}$, with 30s intervals and 120s intervals between eyes open and eyes closed respectively. Measurements were taken in dim light with fixation on a cross or light during periods when the eyes are open. Raw voltage signals at each channel were then bandpass filtered between 2 and $50 \mathrm{~Hz}$ to remove artifacts from motion and electromagnetic interference from power lines respectively (EEGLab suite in Matlab). An ICA was run using a fastICA algorithm in EEGLab, removing artifacts due to eye movements as well as motion artifacts that were not filtered out by the bandpass filter. Frequency spectra of filtered and pruned voltage data was then calculated at 
$500 \mathrm{~ms}$ intervals ( pwelch in Matlab) to match the integration time of fNIRS. Spectral power was then integrated between $7-13 \mathrm{~Hz}$ to determine integrated alpha power and between $20.5-28 \mathrm{~Hz}$ for high beta power. Integrated band powers were then smoothed (Matlab smooth function) and the logarithm of the smoothed power was then plotted against time.
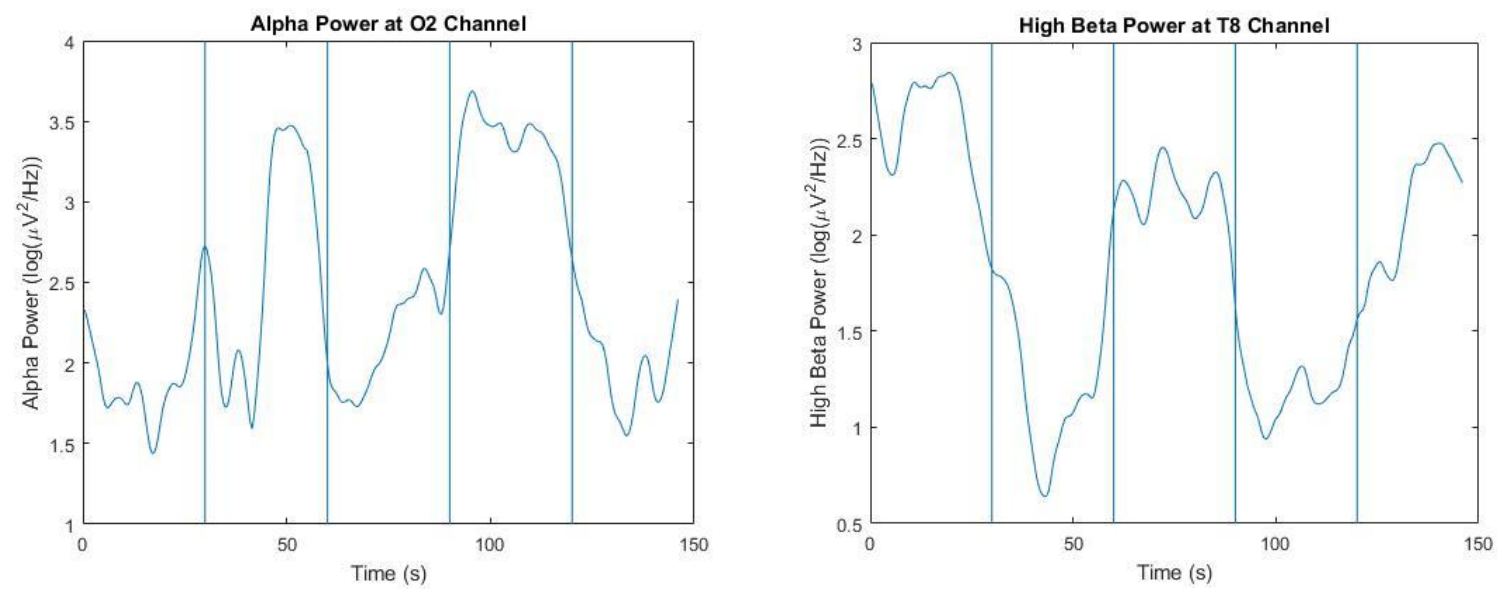

Figure 4.2. Left: Integrated alpha power $(7-13 \mathrm{~Hz})$ of $\mathrm{O} 2$ channel in a subject during an eyes open/eyes closed task. Increased alpha activity is seen in the second and fourth epochs, corresponding to an eyes closed state. Alpha waves are though to originate in the occipital region of the brain. Right: Integrated high beta power $(20.5-28 \mathrm{~Hz})$ of $\mathrm{T} 8$ channel in same subject and task. Beta waves are though to originate in the temporal region.

\subsubsection{Discussion}

As shown in Figure 4.1 the $\mathrm{HbO}_{2}$ and $\mathrm{HHb}$ concentrations show a marked change in response during a breath hold. $\mathrm{HHb}$ concentration decreases and $\mathrm{HbO}_{2}$ concentration increases in response to periods of breath holding and returns back to baseline during normal breathing. This is in agreement with previous studies. [51] 
The integrated alpha power and integrated high beta power show a strong response in subjects, as shown in Figure 4.2. Alpha power increases dramatically when the eyes are closed, especially in the occipital region of the brain. This is in agreement with the fact that the waveform is thought to originate from the occipital region of the brain. For high beta waves, the integrated power was the largest when the eyes were open and decreased when the eyes were closed. This corresponds with beta waves association with concentration and vigilance as the eyes are fixated during periods when the eyes are open.

Preliminary findings using the EEG-fNIRS headset demonstrate that the headpiece is capable of measuring the physiological effects that occur in the brain in both modalities.

\subsection{Developing the Eye Open/Eyes Closed Task as a Prospective Screening Tool for EEG and fNIRS}

\subsubsection{Results}

Knowing that the EEG-fNIRS headset is capable of taking robust measurements of both scalp voltages for EEG and light scattered and absorbed in the brain for fNIRS, finding a task that would show physiological changes in healthy subjects in both EEG and fNIRS signals. The simplest task to test was the eyes open/eyes closed task. In order to determine if the EEG data collected from the eyes open/eyes closed task was significant, time courses from 7 healthy subjects were analyzed. Subjects were recruited from Ryerson University. The data was sampled with simultaneous fNIRS using the method described above in Chapter 4.1 with 120 second intervals between eyes open and eyes closed and alpha and high beta integrated power were 
calculated for each channel. The EEG data was then averaged across all subjects and channels in the EEG cap at the front of the head; AF3, AF4, F7, F8, F3, F4, FC5, and FC6. The logarithm was taken of each individual's average and the average over all subjects and standard deviation were calculated and plotted in Figure 4.3 below.
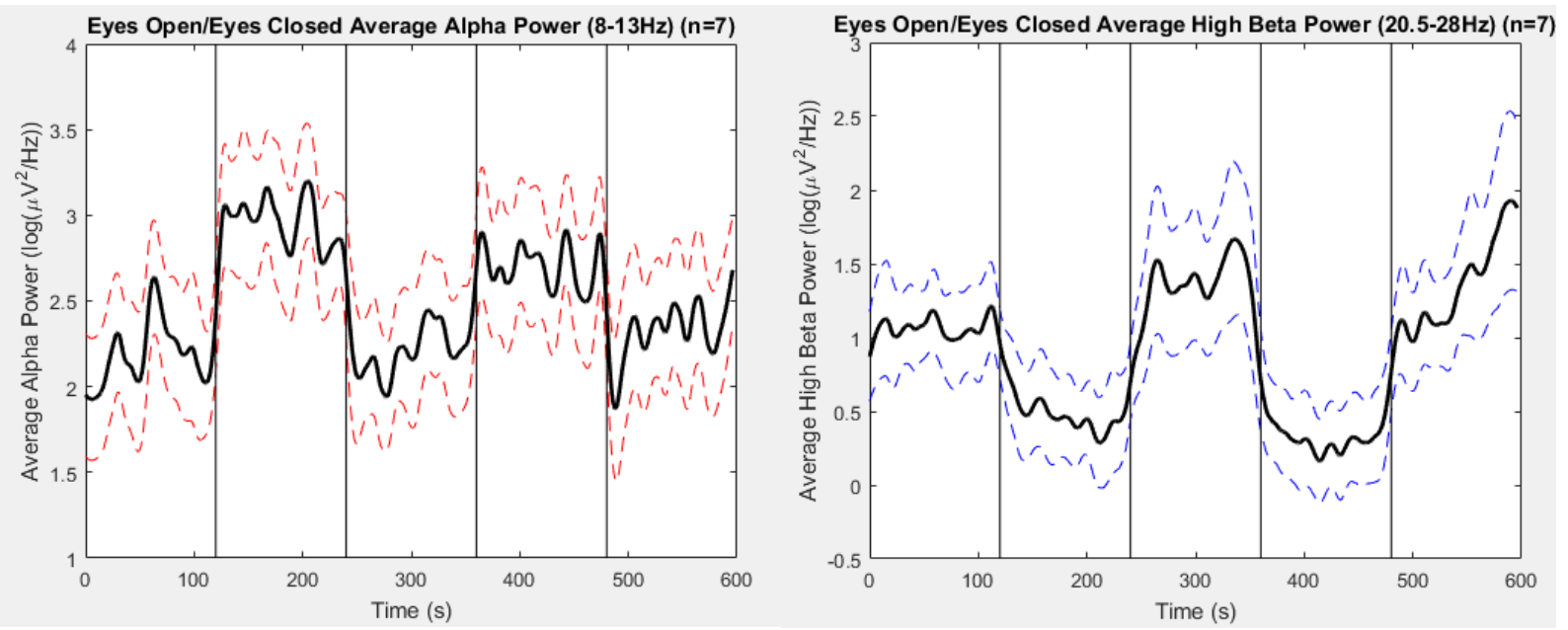

Figure 4.3. Left: Averaged integrated alpha power over frontal channels

from 7 subjects with calculated standard deviation during an eyes open/eyes closed task. Right: Averaged integrated high beta power over frontal channels from the same subjects and task.

To test the significance of the EEG results, the frontal average data of each individual was cut into segments of eyes open and eyes closed. The values of each segment were averaged giving rise to 2 averaged eyes closed and 2 averaged eyes open values for each individual for a total of 14 eyes open averages and 14 eyes closed averages. For eyes open data, the last eyes 
open set was discarded for analysis. Averaged data was then subjected to a 2-sample $t$ test (ttest2 function in Matlab) to test if the samples were significantly different.

The t-test results demonstrated that the integrated alpha powers between eyes open and eyes closed were significantly different, an increase in alpha power by $0.6133 \log \left(\mu \mathrm{V}^{2} / \mathrm{Hz}\right)$, $\mathrm{p}=0.00013$; with a significance level of $\mathrm{p}<0.05$. However, the difference in the integrated high beta power averages between eyes open and eyes closed were not statistically significant, a decrease in integrated high beta power by $0.9275 \log \left(\mu \mathrm{V}^{2} / \mathrm{Hz}\right), \mathrm{p}=0.37$.

A t-test was also performed on individual channels. Time courses for individual channels were pooled across all subjects and the averages alpha and high beta power were compared between eyes open and eyes closed.
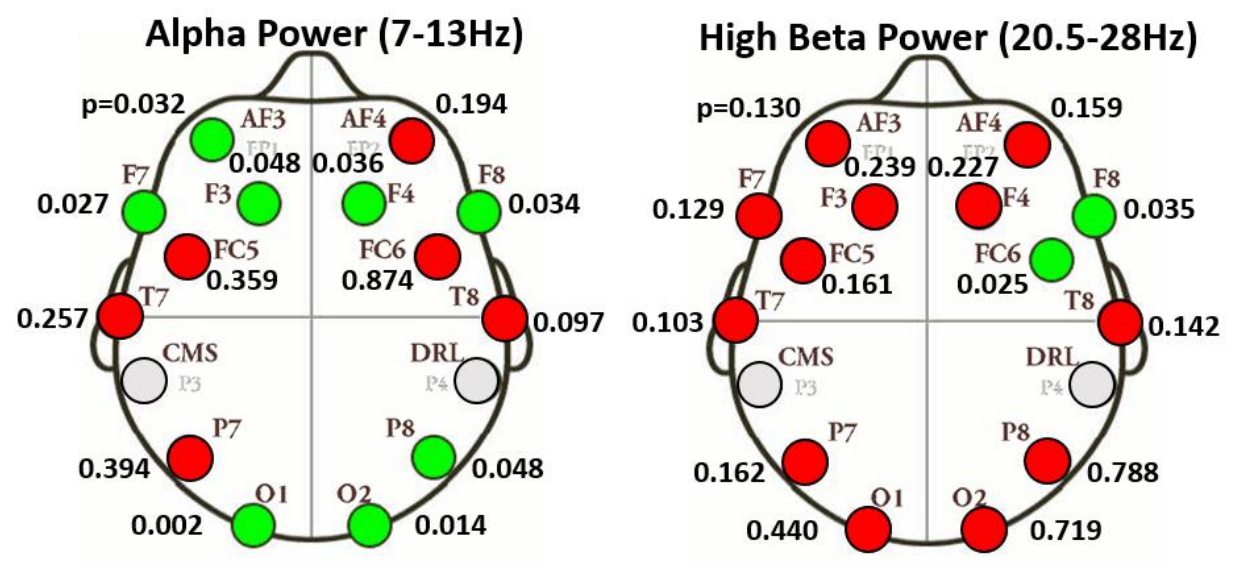

Figure 4.4. T-test results for individual channels for both wavebands used comparing the eyes open against eyes closed state. Channels with significant differences are highlighted. 
The directionality of each subject and channel was also tested to check for consistency of the signal. It was expected that from eyes open to eyes closed, alpha power would increase and high beta power would decrease.

\begin{tabular}{|c|c|c|c|c|c|c|c|c|c|c|c|c|c|c|}
\hline \multirow{2}{*}{ Channel } & \multicolumn{2}{|c|}{ Subject 1} & \multicolumn{2}{|c|}{2} & \multicolumn{2}{|c|}{3} & \multicolumn{2}{|c|}{4} & \multicolumn{2}{|c|}{5} & \multicolumn{2}{|c|}{6} & \multicolumn{2}{|c|}{7} \\
\hline & $\alpha$ & $\beta$ & $\alpha$ & $\beta$ & $\alpha$ & $\beta$ & $\alpha$ & $\beta$ & $\alpha$ & $\beta$ & $\alpha$ & $\beta$ & $\alpha$ & $\beta$ \\
\hline AF3 & $\uparrow$ & $\downarrow$ & $\uparrow$ & $\downarrow$ & $\uparrow$ & $\downarrow$ & $\uparrow$ & $\downarrow$ & $\uparrow$ & $\downarrow$ & $\uparrow$ & $\downarrow$ & $\uparrow$ & $\uparrow$ \\
\hline AF4 & $\uparrow$ & $\downarrow$ & $\uparrow$ & $\downarrow$ & $\uparrow$ & $\downarrow$ & $\uparrow$ & $\downarrow$ & $\uparrow$ & $\downarrow$ & $\uparrow$ & $\downarrow$ & $\uparrow$ & $\uparrow$ \\
\hline F7 & $\uparrow$ & $\downarrow$ & $\uparrow$ & $\downarrow$ & $\uparrow$ & $\downarrow$ & $\uparrow$ & $\downarrow$ & $\uparrow$ & $\downarrow$ & $\uparrow$ & $\downarrow$ & $\uparrow$ & $\downarrow$ \\
\hline F8 & $\uparrow$ & $\downarrow$ & $\uparrow$ & $\uparrow$ & $\uparrow$ & $\downarrow$ & $\uparrow$ & $\downarrow$ & $\uparrow$ & $\downarrow$ & $\uparrow$ & $\downarrow$ & $\uparrow$ & $\downarrow$ \\
\hline F3 & $\uparrow$ & $\downarrow$ & $\uparrow$ & $\uparrow$ & $\uparrow$ & $\downarrow$ & $\uparrow$ & $\downarrow$ & $\uparrow$ & $\downarrow$ & $\uparrow$ & $\downarrow$ & $\uparrow$ & $\uparrow$ \\
\hline F4 & $\uparrow$ & $\downarrow$ & $\uparrow$ & $\uparrow$ & $\uparrow$ & $\downarrow$ & $\uparrow$ & $\downarrow$ & $\uparrow$ & $\downarrow$ & $\uparrow$ & $\downarrow$ & $\uparrow$ & $\uparrow$ \\
\hline FC5 & $\uparrow$ & $\downarrow$ & $\downarrow$ & $\downarrow$ & $\downarrow$ & $\downarrow$ & $\uparrow$ & $\downarrow$ & $\uparrow$ & $\downarrow$ & $\downarrow$ & $\uparrow$ & $\uparrow$ & $\downarrow$ \\
\hline FC6 & $\uparrow$ & $\downarrow$ & $\downarrow$ & $\downarrow$ & $\uparrow$ & $\downarrow$ & $\uparrow$ & $\downarrow$ & $\uparrow$ & $\downarrow$ & $\downarrow$ & $\downarrow$ & $\uparrow$ & $\uparrow$ \\
\hline $\mathrm{T} 7$ & T & $\downarrow$ & $\uparrow$ & $\uparrow$ & $\downarrow$ & $\downarrow$ & $\uparrow$ & $\downarrow$ & $\uparrow$ & $\downarrow$ & $\uparrow$ & $\downarrow$ & $\uparrow$ & $\downarrow$ \\
\hline T8 & $\uparrow$ & $\downarrow$ & $\uparrow$ & $\uparrow$ & $\uparrow$ & $\downarrow$ & $\uparrow$ & $\downarrow$ & $\uparrow$ & $\downarrow$ & $\uparrow$ & $\downarrow$ & $\uparrow$ & $\uparrow$ \\
\hline P7 & $\downarrow$ & $\downarrow$ & $\uparrow$ & $\uparrow$ & $\uparrow$ & $\uparrow$ & $\uparrow$ & $\downarrow$ & $\uparrow$ & $\downarrow$ & $\uparrow$ & $\downarrow$ & $\uparrow$ & $\uparrow$ \\
\hline P8 & $\uparrow$ & $\downarrow$ & $\uparrow$ & $\uparrow$ & $\uparrow$ & $\downarrow$ & $\uparrow$ & $\downarrow$ & $\uparrow$ & $\downarrow$ & $\uparrow$ & $\downarrow$ & $\uparrow$ & $\uparrow$ \\
\hline $\mathrm{O} 1$ & $\uparrow$ & $\downarrow$ & $\uparrow$ & $\uparrow$ & $\uparrow$ & $\downarrow$ & $\uparrow$ & $\downarrow$ & $\uparrow$ & $\downarrow$ & $\uparrow$ & $\downarrow$ & $\uparrow$ & $\uparrow$ \\
\hline $\mathrm{O} 2$ & $\uparrow$ & $\downarrow$ & $\uparrow$ & $\uparrow$ & $\uparrow$ & $\uparrow$ & $\uparrow$ & $\downarrow$ & $\uparrow$ & $\uparrow$ & $\uparrow$ & $\downarrow$ & $\uparrow$ & $\uparrow$ \\
\hline
\end{tabular}

Table 4.1. Directionality of averaged integrated power from transitions from eyes open to eyes closed. Alpha power increased in general and high beta power decreased in general.

In order to determine the effects of the eyes open/eyes closed task on chromophore concentrations in the fNIRS signal, data from 4 subjects were collected and averaged from the same group used for EEG analysis. Three of the subjects were omitted due to using a different spectrometer to collect fNIRS data. Calculated chromophore concentrations for oxyhemoglobin and deoxyhemoglobin were averaged and standard deviation was calculated across the 4 subjects. 

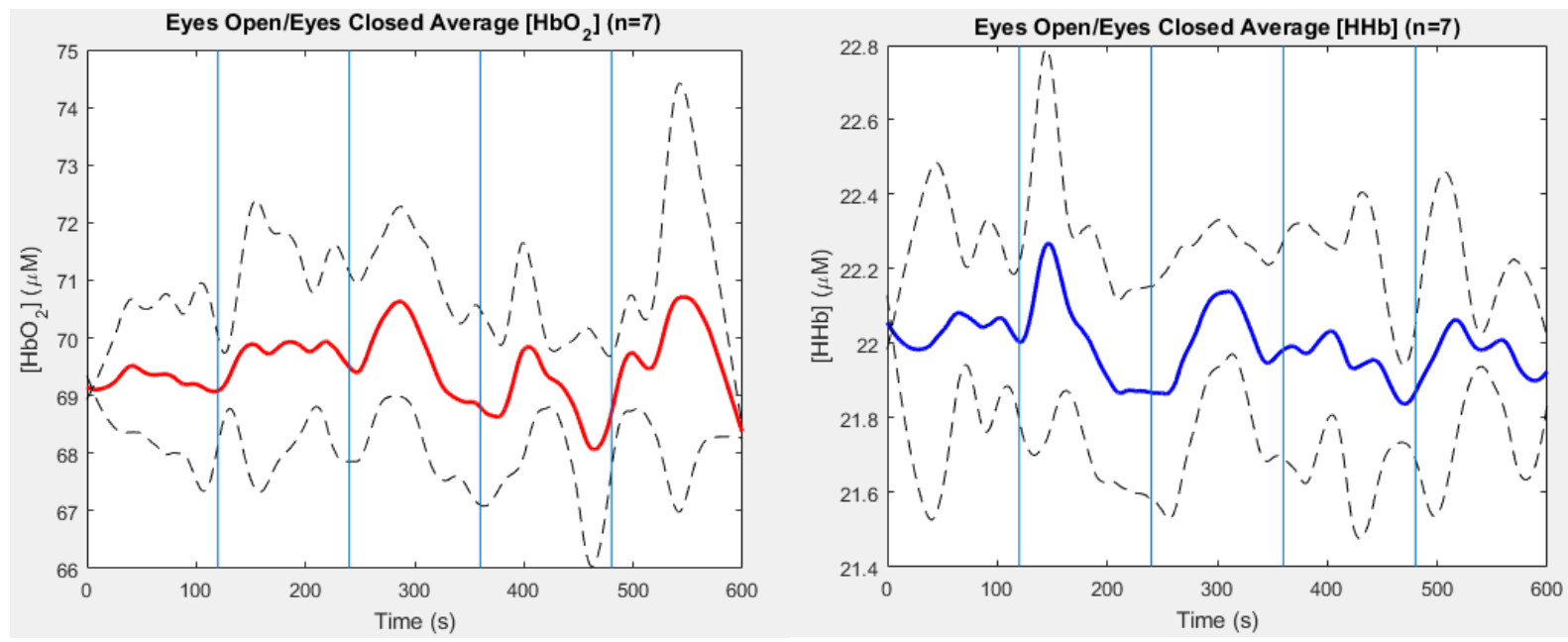

Figure 4.5. Left: Averaged oxyhemoglobin concentrations across 4

subjects for the eyes open/eyes closed task. Right: Averaged

deoxyhemoglobin concentrations for the eyes open/eyes closed task.

Standard deviation was calculated for both chromophores.

\subsubsection{Discussion}

From Figure 4.3, one can see that the integrated alpha power increases by around a factor between eyes open and eyes closed. Integrated high beta power also decreases by around 0.5-1 factors between eyes open and eyes closed. Combined with the directionalities in both integrated alpha and high beta power between eyes open and eyes closed conditions, as shown in Table 4.1, on average, alpha power increases when a subject's eyes are closed as opposed to when the eyes are open and the opposite effect is true for high beta power, which decreases when the eyes are closed.

It was found that across the frontal channels, integrated alpha power showed a significant increase in power, $0.6133 \log \left(\mu \mathrm{V}^{2} / \mathrm{Hz}\right), \mathrm{p}=0.00013$, while the integrated high beta power did not 
show a significant decrease in power, $\log \left(\mu \mathrm{V}^{2} / \mathrm{Hz}\right), \mathrm{p}=0.37$. This may be due to the larger variance in high beta powers across subjects, especially when the eyes are open. This could be due to factors such as the large age range between subjects, as absolute band powers change in aging populations. [52] It is also likely that more subjects are needed for the high beta powers to be statistically significant, especially to narrow the large variance in the high beta power data.

It is clear from Figure 4.4, that the changes in integrated alpha power are statistically significant across the majority of the channels in the frontal region of the brain, lending to good statistical significance of the averaged data. Surprisingly, the integrated high beta power did not show statistical significance across most of the channels, with better p-values in the temporal and frontal regions than the parietal and occipital regions overall which is expected due to the waves are thought to be generated in the temporal region of the brain. Likewise, the strongest difference in the integrated alpha power between the eyes open and eyes closed condition were found in the occipital region at the back of the head where it is thought that alpha waves originate. In terms of utilizing EEG as a screening tool and the simple eyes open/eyes closed task as a test for brain injury, using alpha power would serve as a better means for measuring EEG signal than high beta power as it provides a more robust difference in signal between the eyes open and eyes closed condition.

In terms of fNIRS, the data shows a large spread of values in both oxyhemoglobin and deoxyhemoglobin, such that the standard deviation is much larger than the change in the signal across transitions between eyes open and eyes closed. Ignoring the large standard deviations, one can see that there seems to be an increase in both oxyhemoglobin and deoxyhemoglobin as a response to a transition between an eyes open and eyes closed state from both eyes open to eyes 
closed and vice versa. This would suggest that total hemoglobin levels respond to the change between the two states. Whether this is due to the physiological change in neural activity is yet to be determined. It is important to note that though there seems to be a pattern in the fNIRS data for the eyes open/eyes closed task, as of yet, the averaged data is not statistically significant as the changes in the concentration levels are smaller than the standard deviation across 4 subjects. More work is needed before a robust conclusion can be drawn.

Other works using the eyes open/eyes closed task include fMRI studies, such as the study conducted by Marx et al. [56] It was found that brain activation increased when the eyes were open and decreased when the eyes were closed using similar conditions to this study. The fMRI data present by Marx shows the opposite behaviour of the alpha power behaviour we have found in this study. This is in agreement with studies that show fMRI brain activation is negatively correlated with alpha power measured through EEG. [55] 


\subsection{Testing EEG for Trail Making Task}

\subsubsection{Results}

To further test the robustness of the EEG headset, several additional tasks were performed with the combined EEG-fNIRS headset; a clock drawing task, letter cancellation task and a trail making task. Of the additional tests done using the headset, the trail making task was selected as a candidate for further testing because of the number of studies conducted using the task at St. Michael's Hospital for fMRI studies. The trail making task would have the most comparable data to tasks already completed in previous studies. [53]

The trail making task was performed in conjunction with an eyes open/ eye closed task with the order of the two tasks randomized to minimize the effects of fatigue from the data set.

Data was collected from 11 subjects recruited from Ryerson University. Subjects were not trained before taking the test. The task performed was detailed in Chapter 3.4 and was performed in dim light settings. Data collected was averaged over the frontal channels (same as the eyes open/eyes closed task) of all 11 subjects for a total of 88 channels contributing to the frontal average. The logarithm was taken of the integrated alpha and high beta powers and the average and standard deviations of the frontal channels were calculated and plotted in Figure X below. 

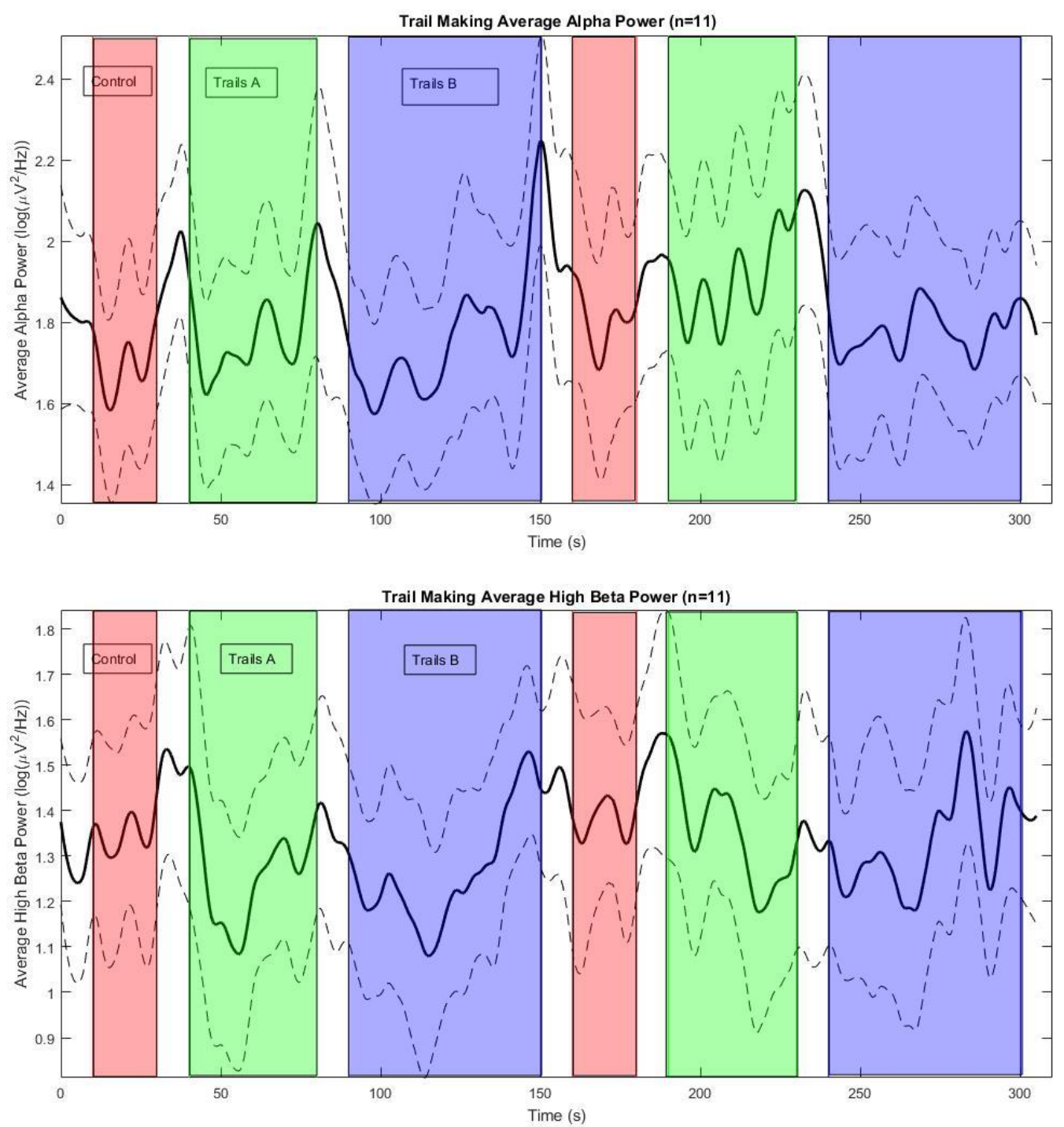

Figure 4.6 Top: Averaged integrated alpha power for trail making across

11 subjects. Bottom: Averaged integrated high beta power for trail

making task. Standard deviation was calculated for both waveband

powers and control, trails A and trails B sections of the task are

highlighted. 


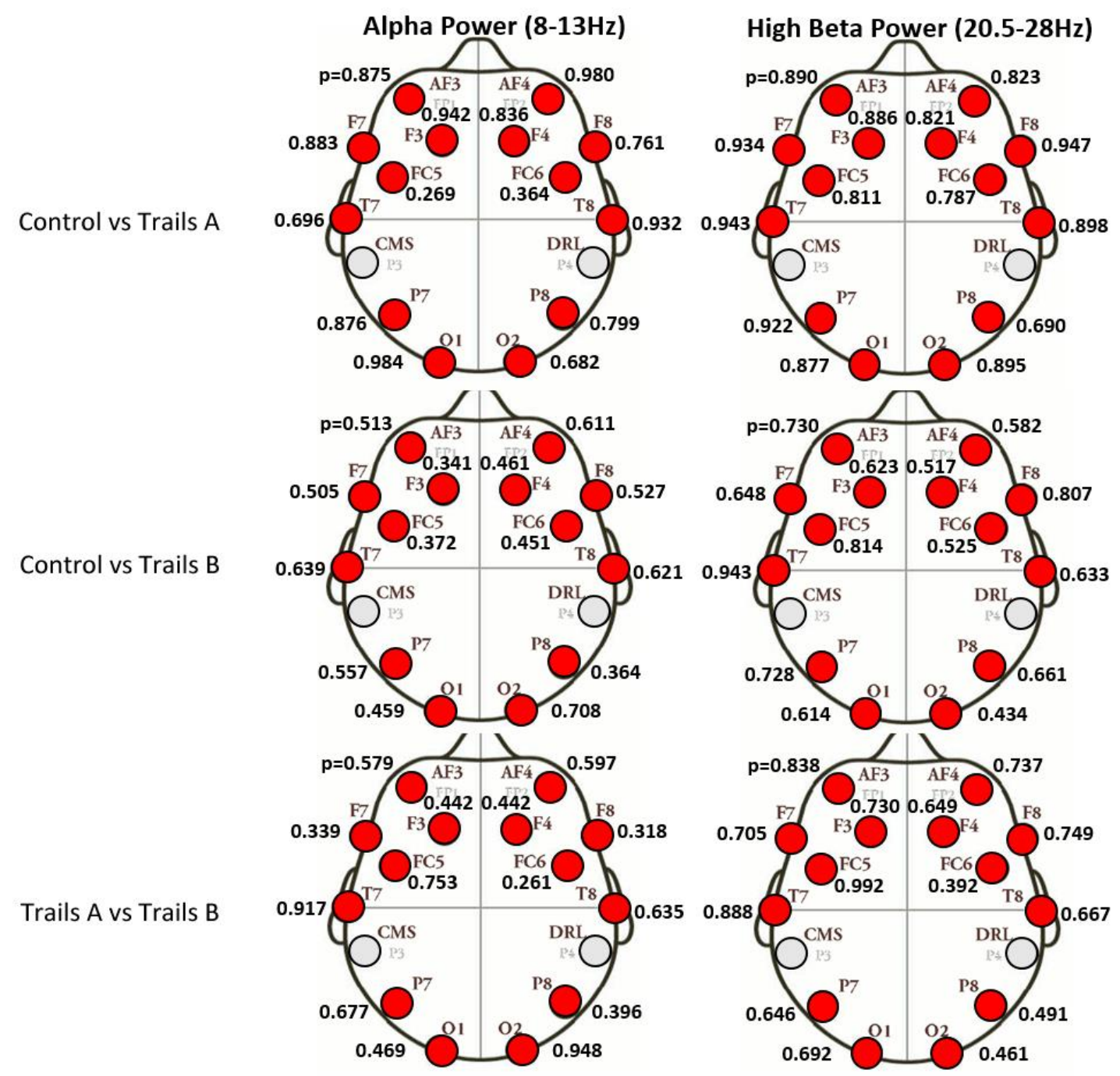

Figure 4.7 T-test results for individual channels comparing averaged values in control, trails A and trails B. No channels showed significant differences between averaged integrated power.

An initial test to determine the significance of the data collected through EEG for the trail making task was to compare the average values of the integrated powers over the various trails; compare the average value during the control to trails A, compare the average power values during the control and trails $\mathrm{B}$, and compare the average integrated power during trails $\mathrm{A}$ and 
trails B. To do this, the integrated powers, both alpha and high beta over individual channels, were averaged over each of the control, trails A and trails B sections of the task. At each channel a 2-sample t-test was performed between the average values of control and trails A, control and trails B, and trails A and trails B.

A 2-sample t-test was also performed on the averaged frontal data and it was found that between the control and trails A there was an increase in integrated alpha power by 0.2278 $\log \left(\mu \mathrm{V}^{2} / \mathrm{Hz}\right), \mathrm{p}=0.6305$ and high beta power decreased by $0.1478 \log \left(\mu \mathrm{V}^{2} / \mathrm{Hz}\right), \mathrm{p}=0.7851$. Between the control and trails $\mathrm{B}$, there was an increase in alpha power by $0.1945 \log \left(\mu \mathrm{V}^{2} / \mathrm{Hz}\right)$, $\mathrm{p}=0.8564$, and a $0.4902 \log \left(\mu \mathrm{V}^{2} / \mathrm{Hz}\right)$ decrease in high beta power, $\mathrm{p}=0.5626$. Between trails $\mathrm{A}$ and trails $\mathrm{B}$, there was a decrease in alpha power by $0.0333 \log \left(\mu \mathrm{V}^{2} / \mathrm{Hz}\right), \mathrm{p}=0.7388$, and a 0.3424 $\log \left(\mu \mathrm{V}^{2} / \mathrm{Hz}\right)$ decrease in high beta power, $\mathrm{p}=0.7713$.

None of the results from taking the average values over the control, trails A or trials B resulted in statistically significant results. Rather than sample the average values over each of the segments, it is shown from Figure $\mathrm{X}$ that the integrated power changes during trails $\mathrm{A}$ and trails B from early in the trail, when the subject is engaged in the task, to late, when the task has likely been completed. In order to test if there was a difference in the integrated powers when the subject was engaged versus when the subject was at rest, a 5 second window was taken around the minimum in the averaged early trails A and trails B for each subject and a 5 second window was taken during the period of rest after the trails had ended. Rather than compare individual channels for statistical significance, the channels were pooled together and a 2-sample t-test was performed over all frontal channels between early trails A and rest, and early trails B and rest. Early trails A and trails B data was also compared to rest periods before the task. In addition to performing a t-test on frontal channels, the frontal channels were also split into front-right and 
front-left and a 2-sample t-test was performed using these divisions as well. Rather than pool the first and second trail of the same type in each run, the first and second runs were kept separate to determine if there were effects due to fatigue or learning.

\begin{tabular}{ccccccc}
\hline \hline Trail 1 & $\begin{array}{c}\text { Frontal } \alpha \\
\left(\log \left(\mu \mathrm{V}^{2} / \mathrm{Hz}\right)\right)\end{array}$ & Frontal $\beta$ & $\begin{array}{c}\text { Front-Left } \\
\alpha\end{array}$ & $\begin{array}{c}\text { Front-Left } \\
\beta\end{array}$ & $\begin{array}{c}\text { Front- } \\
\text { Right } \alpha\end{array}$ & $\begin{array}{c}\text { Front- } \\
\text { Right } \beta\end{array}$ \\
\hline $\begin{array}{c}\text { Rest } \\
\text { Before- }\end{array}$ & -0.312, & -0.391, & -0.244, & -0.402, & -0.416, & -0.547, \\
Trail A & $\mathrm{p}=0.040$ & $\mathrm{p}=0.544$ & $\mathrm{p}=0.034$ & $\mathrm{p}=0.306$ & $\mathrm{p}=0.055$ & $\mathrm{p}=0.793$ \\
Rest & -0.341, & -0.259, & -0.208, & -0.264, & -0.342, & -0.476, \\
Before- & $\mathrm{p}=0.045$ & $\mathrm{p}=0.450$ & $\mathrm{p}=0.039$ & $\mathrm{p}=0.489$ & $\mathrm{p}=0.039$ & $\mathrm{p}=0.422$ \\
Trail B & & & & & \\
Trail A- & 0.344, & 0.114, & 0.293, & 0.4022, & 0.314, & 0.480, \\
Rest After & $\mathrm{p}=0.073$ & $\mathrm{p}=0.804$ & $\mathrm{p}=0.116$ & $\mathrm{p}=0.518$ & $\mathrm{p}=0.067$ & $\mathrm{p}=0.953$ \\
Trail B- & 0.479, & 0.405, & 0.468, & 0.422, & 0.510, & 0.627, \\
Rest After & $\mathrm{P}=0.009$ & $\mathrm{p}=0.305$ & $\mathrm{p}=0.012$ & $\mathrm{p}=0.268$ & $\mathrm{p}=0.010$ & $\mathrm{p}=0.0362$ \\
\hline \hline Trail 2 & $\mathrm{Frontal} \alpha$ & Frontal $\beta$ & Front-Left & Front-Left & Front- & Front- \\
& $\left(\log \left(\mu \mathrm{V}^{2} / \mathrm{Hz}\right)\right)$ & & $\alpha$ & $\beta$ & Right $\alpha$ & Right $\beta$ \\
\hline Rest & -0.113, & -0.301, & 0.159, & -0.334, & -0.132, & -0.344, \\
Before- & $\mathrm{p}=0.234$ & $\mathrm{p}=0.547$ & $\mathrm{p}=0.191$ & $\mathrm{p}=0.482$ & $\mathrm{p}=0.302$ & $\mathrm{p}=0.628$ \\
Trail A & & & & & & \\
Rest & -0.392, & -0.238, & -0.438, & -0.266, & -0.442, & -0.287, \\
Before- & $\mathrm{p}=0.039$ & $\mathrm{p}=0.492$ & $\mathrm{p}=0.037$ & $\mathrm{p}=0.0651$ & $\mathrm{p}=0.047$ & $\mathrm{p}=0.402$ \\
Trail B & & & & & \\
Trail A- & 0.352, & 0.185, & 0.391, & 0.192, & 0.316, & 0.283, \\
Rest After & $\mathrm{p}=0.060$ & $\mathrm{p}=0.801$ & $\mathrm{p}=0.049$ & $\mathrm{p}=0.768$ & $\mathrm{p}=0.086$ & $\mathrm{p}=0.837$ \\
Trail B- & 0.265, & 0.381, & 0.347, & 0.327, & 0.692, & 0.624, \\
Rest After & $\mathrm{p}=0.452$ & $\mathrm{p}=0.612$ & $\mathrm{p}=0.251$ & $\mathrm{p}=0.443$ & $\mathrm{p}=0.695$ & $\mathrm{p}=0.794$ \\
\hline \hline
\end{tabular}

Table 4.2. Calculated average differences between rest and trails A and B separated before and after the trails and between the first and second runs. Significant differences are highlighted, $\mathrm{p}<0.05$. 
Trails A
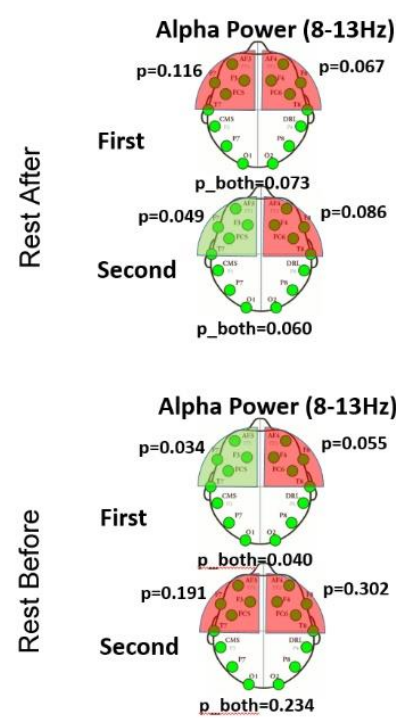
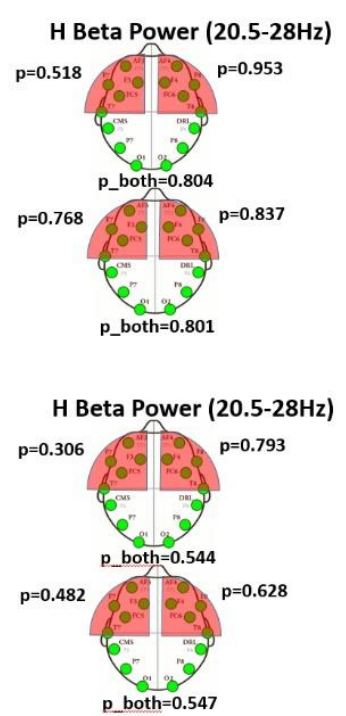

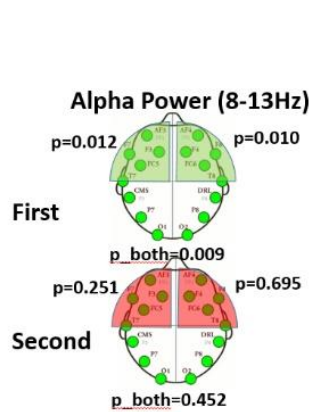

Trails B

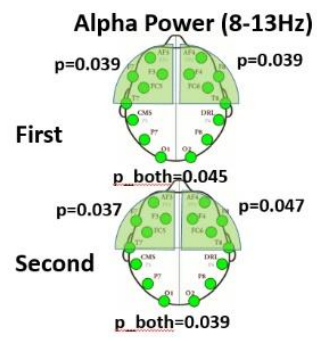

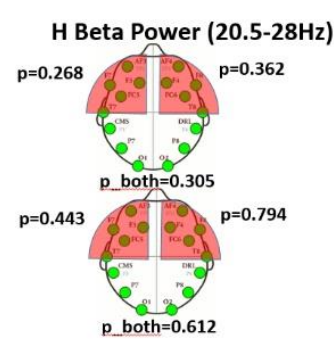

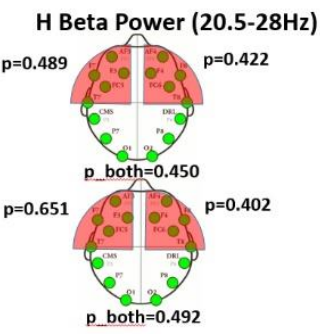

Figure 4.8 T-test results for comparing trails $\mathrm{A}$ and $\mathrm{B}$ to rest

periods directly before and after the trails task during a trail making task.

T-test results are shown for front-left, front-right and frontal averages for both the first and second runs of each trail.

\subsubsection{Discussion}

From Figure 4.6, it is shown that the integrated waveband powers change as a function of time during the trails tasks. At the start of a trails task, the alpha power decreases and slowly increases as the trails task is completed. This may be explained by the fact that the brain is engaged during the initial phase of the trails task with planning. As the subjects completed the task, alpha power increased as the brain is no longer engaged. Interestingly, high beta power also decreased in response to the presentation of the trails and slowly increased over the course of the trail. This may be due to beta waves involvement in vigilance. [44] Beta waves are also 
associated with concentration and muscle tenseness, as subjects finish the task, they may be more concentrated on not moving, increasing beta power. The spread of integrated high beta powers was quite large, resulting in poor results when statistical analyses were performed.

Initial attempts to perform statistical analyses on the data was not successful. Averages of each of the tasks within a trail making session did not yield statistically significant results. This method was initially chosen because it matched the protocols used in fMRI studies performed at St. Michael's Hospital, from which the trail making task was adapted from. Statistically relevant results would have been comparable to data already collected in fMRI studies. From Figure 4.6, it is clear that the integrated powers in both alpha and high beta bands do not stay flat during the trails tasks. In order to better understand the response of the EEG signal to the trail making task, smaller segments of the time course were chosen, comparing periods of rest where the subject is fixated on a cross, to when the subject is first presented with the trail.

This method yielded the best results. Significant differences in frontal alpha powers were found between periods of rest and initial presentation of the trails in both trails $\mathrm{A}$, the less difficult task, and trails B, the more difficult task. Table 4.2 shows that alpha power decreases upon initial presentation of the trail and recovers afterwards. Table 4.2 and Figure 4.8 also demonstrates that the effects are more dramatic in the first run of trails than the second; 3 of the 4 integrated power differences were significant $(\mathrm{p}<0.05$, and all 4 were within $\mathrm{p}<0.1)$ in frontal averages for the first set of trails while only 1 of the differences calculated was significant ( 2 were within $\mathrm{p}<0.1$ ). This may be due to fatigue or the effects of learning. The latter being detrimental, as it means subsequent trials using the same subject may yield worse results.

It was shown in Table 4.2 that integrated high beta power did not yield any statistically significant results, even though the differences between the rest state and initial presentation of 
the trails was larger in some cases when compared to alpha power. This is due to the relatively large variance in the values for high beta power, exacerbated by it's comparatively low absolute power.

Previous studies that have used the trail making task include fMRI studies. Such studies tend to focus on frontal lobe function and seek to test a person's ability to scan, track and also test attention. [57] Conventional fMRI studies used the averaged activation over the trails data and test between trails A and trails B (typically trails B minus trails A). Activated sites include the inferior frontal sulcus, middle frontal gyrus, and lower precentral gyrus, all on the left side of the brain. [58] This may correlate with the findings herein for the trail making task. From Table 4.2 and Figure 4.8, frontal-left averaged data showed the most significant differences between rest and trails conditions.

The main advantage of using an eyes open/eyes closed task was that the procedure was the simplest to set up and run. It was also easiest to control variables such as cranial movement and its ease to perform by the subject. However, the trail making task offers some advantages over the eyes open/eyes closed task. The eyes open/eyes closed task primarily shows the greatest difference in the occipital region of the brain. [59] This activation is also seen in fMRI studies. [56] However, measurements using the EEG-fNIRS headset takes fNIRS measurements at the frontal regions of the brain around F7 and F8. The trail making task shows activation in the brain closer to front. [57]

In developing a potential screening tool for brain injuries and diseases, it would seem that the data collected from the eyes open/eyes closed and trail making task would be robust enough, at least using EEG. More work would be needed in determining how to implement fNIRS for 
more statistically viable results, as well as hypothesize how an injured or diseased brain would differ from the healthy brains measured. 


\section{Conclusion}

\subsection{Summary of Results and Conclusions}

In this work, we have developed an electroencephalography-functional near infrared spectrometry (EEG-fNIRS) attachment to an Emotiv EPOC+ EEG headset capable of performing simultaneous measurements of scalp voltage and the absorption of light through the head for use as a screening tool for brain trauma and disease.

The first step in testing the EEG-fNIRS attachment was to determine if the signals measured from each modality produced measurements that aligned with previous results. It was demonstrated that the fNIRS data matched expected behaviour for a breath hold task.

Deoxyhemoglobin levels were shown to decrease in a subject during the breath hold task while oxyhemoglobin, and subsequently total hemoglobin increased during a breath hold, matching previous studies. EEG measurements were verified using an eyes open/eyes closed task and integrated alpha $(7-13 \mathrm{~Hz})$ and high beta $(20.5-28 \mathrm{~Hz})$ powers were analyzed. Alpha power was shown to increase when the eyes were closed and high beta power was shown to decrease when eyes were closed.

After verifying that the headset was capable of producing reliable measurements, data obtained from the eyes open/eyes closed tasks were analyzed. Alpha power and high beta power time courses for the task were averaged over the frontal channels and across all subjects. A 2sample t-test was performed on average eyes open power against average eyes closed power. The average change in alpha power was found to be an increase of $0.6133 \log \left(\mu \mathrm{V}^{2} / \mathrm{Hz}\right), \mathrm{p}=0.00013$, from eyes open to eyes closed. High beta power was found to not be statistically significant, but decreased by $0.9275 \log \left(\mu \mathrm{V}^{2} / \mathrm{Hz}\right), \mathrm{p}=0.37$. 
A statistical analysis revealed that not only was the averaged data not significant for high beta power, but the majority of individual channels were also no statistically significant. Changes in alpha power between the eyes open and eyes closed state was statistically significant across the majority of channels, with 6 of the 8 frontal channels showing significant changes in alpha power between the two states. Analyzing the directionality of individual channels agreed with averaged data in that alpha power increases with eyes closed and high beta power decreases with eyes closed.

Functional NIRS data was taken simultaneously alongside the EEG data and the averaged data was found to have a large standard deviation with respect to the changes in the signal. It was obvious that data collect from such a small data set would not yield statistically relevant results, however, from just 4 subjects that took measurements using the same spectrometer, a wave-like pattern does seem to be present in the averaged chromophore concentrations. Additional measurements of fNIRS would need to be taken.

From the results of the eyes open/eyes closed task, the task itself provides robust data in EEG when taking integrated alpha power measurements between the two states. The eyes open/eyes closed task is an ideal candidate for use as a screening tool because of its simplicity; motion artifacting can be reduced due to no extraneous movements needed and it is simple enough that a wide range of subjects can be analyzed, as opposed to some tasks that may be too difficult for children or the elderly to grasp. Additionally, the eyes open/eyes closed task has also been used in applications outside of EEG, such as fMRI.

Trail making data was measured using the EEG-fNIRS headset. Integrated alpha power and high beta power were analyzed. The average frontal powers were plotted and revealed that 
both powers decreased upon onset of a trails being presented and recovered over the course of the trails.

Trail making data was then analyzed to produce more robust results. Rather than analyze the data channel by channel, the data was split into frontal, front-left and front-right to produce averaged results. Results were also split between the first and second run to reduce the effects of fatigue and learning. Instead of measuring the averaged data, a 5 second window of when the waveband power was minimized during the trails task was compared to periods of rest directly before and after the trail. It was found that the there was a significant change in the integrated alpha power between the periods of rest and during the trails.

From the data collected, the most promising avenue for the application of the EEG-fNIRS headset would be to use an eyes open/eyes closed task and measure the difference between the alpha power when the eyes are open and the eyes are shut. Additionally, a trail making task can be performed, analyzing the frontal data, and measuring alpha power early in the trails and comparing it to alpha power during the rest before and after the trail. The advantages of each type of task was discussed. Additional work is needed in order to get statistically relevant data from fNIRS to be incorporated.

Several limitations were present in the study. NIRS placement of optodes were limited to the frontal region to avoid hair. Studies were attempted in the occipital region of the head to correspond with the strength of the alpha waves in this region as well as match fMRI data for eyes open/eyes closed tasks, however it was found that the hair severely limited the signals that were able to be measured at the back of the head. This effect was particularly exaggerated in individuals with dark hair as the melanin present in the hair shaft and follicles embedded in the scalp acted as strong absorbers of light in the NIR region. Another limitation concerning NIRS 
was the low signal measured on the forehead. One way to rectify the low signal would have been to increase the integration time, however this would reduce the temporal resolution.

Limitations in the EEG was a rather poor spatial resolution of the Emotiv EPOC+ headset (only 14 channels). However, more robust EEG headcaps, say a 100-channel EEG headcap, are far more expensive and much more difficult to set up. Since the ultimate goal of this study was to produce a simple and inexpensive alternative, using an EEG cap with more electrodes would defeat the purpose of the study.

\subsection{Future Work}

One severe limitation of the study was the small amount of fNIRS data collected in comparison. In the future, it may be an option to collect NIRS data at sites other than just F7 and F8. However, in order to do this, it may be necessary to either shave the head at other sites and use a light source specifically built for the NIR region as excessive light in the visible region is absorbed by the skin and hair, which may cause burns before a usable signal is obtained in fNIRS. fNIRS can also be collected at more sites in the prefrontal region using multiple detection sites. Additionally, NIRS data would benefit greatly from having a larger pool of participants to draw from in order to get statistically relevant data out of the measurements taken.

A simple study that may be performed in the future may be to test a similar commercially available EEG headset. As these headsets are relatively inexpensive, it is reasonable to expect that each type of a headset may have some 'quirks' with respect to a proper EEG headcap meant for clinical settings. Testing the results gathered herein with other cheap headsets would further validate the methods used in this work. 
The algorithms used to filter and clean the data was done manually. One possible avenue for further research would be to automate the process in which artifacts such as eye-blink artifacts are removed. It would likely require a learning algorithm to teach the program to recognize eye blinks in EEG data.

Finally, the ultimate goal of this study would be move on from healthy controls as subjects of the study to diseased and/or victims of brain trauma. Hypotheses would need to be developed and tested. It is expected that for concussed patients, the difference between the eyes open and eyes closed state would be smaller. Elevated alpha power have been seen in previous studies. In addition to expanding the study to concussed patients, the EEG-fNIRS data can also be combined with fMRI studies. Studies have already been conducted using fMRI as a singular modality for eyes open/eyes closed $[54,56]$ and trail making tasks already. Previous studies have also found that EEG alpha power is negatively correlated with MR signals. [55] Other tasks that can be analyzed are clock drawing and letter cancellations tasks that were performed with the EEG-fNIRS headset, but were not analyzed extensively. By incorporating other established modalities, the robustness of the EEG-fNIRS headset can be improved for clinical use.

Many of the tasks used in this project are physiological in nature. More studies using cognitive tasks; as reduced cognitive function is a hallmark of mTBIs; such as the clock-drawing and letter cancellation tasks. Another task that is currently being used in the n-back test in which subjects are requested to name letters ' $n$ ' positions back in a sequence. This has shown promise in fNIRS [66], but may prove challenging for EEG which is susceptible to the movement of the tongue. 


\section{References}

[1] I. R. Casson, D. C. Viano, J. W. Powell, E. J. Pellmen, "Twelve Years of National Football League Concussion Data," Sports Health, 2(6): 471-483 (2010).

[2] P. McCrory, W. H. Meeuwisse, M. Aubry, B. Cantu, J. Dvorak, R. J. Echemendia, L. Engebresten, K. Johnston, J. S. Kutcher, M. Raftery, A. Sills, B. W. Benson, G. A. Davis, R. G. Ellenbogen, K. Guskiewicz, S. A. Herring, G. L. Iverson, B. D. Jordan, J. Kissick, M. McCrea, A. S. McIntosh, D. Maddocks, M. Makdissi, L. Purcell, M. Putukian, K. Schneider, C. H. Tator, M. Turner, "Consensus statement on concussion in sport: the 4th International Conference on Concussion in Sport held in Zurich, November 2012,” Br J Sports Med, 47: 250-258 (2013).

[3] L. J. Stovner, H. Schrader, D. Mickeviciene, D. Surkiene, T. Sand, "Headache after concussion," European Journal of Neurology, 16: 112-120 (2009).

[4] K. G. Harmon, J. A. Drezner, M. Gammons, K. M. Guskiwicz, M. Halstead, S. A. Herring, J. S. Kutcher, A. Pana, M. Putukian, W. O. Roberts, “American Medical Society for Sports Medicine position statement: concussion in sport,” Br J Sports Med, 47: 15-26 (2013).

[5] K. M. Guskiewicz, J. Register-Mihalik, P. McCrory, M. McCrea, K. Johnston, M. Makdissi, J. Dvorak, G. Davis, W. Meeuwisse, "Evidence-based approach to revising the SCAT2: Introducing SCAT3.," Br J Sports Med, 47(5): 289-293 (2013). 
[6] D. Ellemberg, L. C. Henry, S. N. Macciocchi, K. M. Guskiewicz, S. P. Broglio, “Advances in sport concussion assessment: from behavioural to brain imaging measures," Journal of Neurotrauma, 26: 2365-2382 (2009).

[7] G. T. Herman, "Fundamentals of computerized tomography: Image reconstruction from projection, 2nd edition," Springer (2009).

[8] W. J. Schmepp, "Magnetic Resonance Imaging: Mathematical Foundations and Applications," Wiley (1998).

[9] I. Linfante, R. H. Llinas, L. R. Caplan, S. Warach, “MRI Features of Intracerebral Hemorrhage Within 2 Hours From Symptom Onset,” Stroke, 30: 2263-2267 (1999).

[10] C. S. Kidwell, J. A. Chalela, J. L. Saver, S. Starkman, M. D. Hill, A. M. Demchuk, J. A. Butman, N. Patronas, J. R. Alger, L. L. Latour, M. L. Luby, A. E. Baird, M. C. Leary, M. Tremwel, B. Ovbiagele, A. Fredieu, S. Suzuki, J. P. Villablanca, S. Davis, B. Dunn, J. W. Todd, M. A. Ezzeddine, J. Haymore, J. K. Lynch, L. Davis, S. Warach, "Comparison of MRI and CT for Detection of Acute Intracerebral Hemorrhage," JAMA 292(15): 18231830 (2004).

[11] K. R. Byrnes, C. M. Wilson, F. Brabazon, R. von Leden, J. S. Jurgens, T. R. Oakes, R. G. Selwyn, "FDG-PET imaging in mild traumatic brain injury: a critical review," Front Neuroenergetics 5(13): 1-24 (2014).

[12] “Fee Schedule," Canada Diagnostic. Accessed: 2016-09-08, url: http://www.canadadiagnostic.com/info/fees/. 
[13] “About CMI’s Rates,” Canadian Magnetic Imaging. Access: 2016-09-08, url: http://www.canmagnetic.com/scans-rates/.

[14] C. Elliot, "Should Medicare Cover the Cost of Brain Imaging Tests for Alzheimer's Diagnosis?” MyBrainTest (2013). Accessed: 2016-09-08, url: http://www.mybraintest.org/2013/07/should-medicare-cover-the-cost-of-brain-imagingtests-for-alzheimers-diagnosis/.

[15] K. Smith, “fMRI 2.0,” Nature, 484: 24-26 (2012).

[16] B. B. Forster, A. L. MacKay, K. P. Whittall, K. A. Kiehl, A. M. Smith, R. D. Hare, P. F. Liddle, "Functional magnetic resonance imaging: the basics of blood-oxygen-level dependent (BOLD) imaging," Can Assoc Radiol J., 49(5): 320-329 (1998).

[17] J. M. Murkin, M. Arango, "Near-infrared spectroscopy as an index of brain and tissue oxygenation," BJA, 103: i3-i13 (2009).

[18] A. F. Jackson, D. J. Bolger, "The neurophysiological bases of EEG and EEG measurement: a review for the rest of us," Psychophysiology, 51(11): 1061-1071 (2014).

[19] “EPOC+," EMOTIV. Accessed: 2016-09-08, url: https://www.emotiv.com/epoc/.

[20] B. Agrell, O. Dehun, “The clock-drawing test," Age and Ageing, 27: 399-403 (1998).

[21] A. M. Smith, M. C. Mandini, S. Nie, "Second window for in vivo imaging," Nat Nanotechnol, 4(11): 710-711 (2009). 
[22] G. E. Strangman, Z. Li, Q. Zhang, "Depth Sensitivity and Source-Detector Separations for Near Infrared Spectroscopy Based on the Colin27 Brain Template,” Plos One, 8(8) (2013).

[23] D. F. Swinehart, “The Beer-Lambert Law,” J. Chem. Educ., 39(7): 333 (1962).

[24] L. Kocsis, P. Herman, A. Eke, “The modified Beer-Lambert law revisited,” Phys Med Biol, 51(5): N91-N98 (2006).

[25] P. Shapley, “Absorbing light with organic molecules," University of Illinois. Accessed: 2016-09-08, url: http://butane.chem.uiuc.edu/pshapley/GenChem2/B2/1.html.

[26] J. D. Johansson, K. Wardell, "Intracerebral quantitative chromophore estimation from reflectance spectra captured during deep brain stimulation implant," J Biophotonics, 6(5): 435-445 (2013).

[27] M. Weissbluth, "Hemoglobin," Molecular Biology Biochemistry and Biophysics, 15: 10-26 (1974).

[28] M. F. Perutz, "Regulation of Oxygen Affinity of Hemoglobin: Influence of Structure of the Globin of the Heme Iron,” Ann Rev Biochem, 48: 327-386 (1979).

[29] B. Chance, J. S. Leigh, H. Miyake, D. S. Smith, S. Nioka, R. Greenfeld, M. Finander, K. Kaufmann, W. Levy, M. Young, “Comparison of time-resolved and -unresolved measurements of deoxyhemoglobin in brain," PNAS, 85(14): 4971-4975 (1988).

[30] C. Ostermeier, S. Iwata, H. Michel, "Cytochrome c oxidase," Current Opinion in Structural Biology, 6(4): 460-466 (1996). 
[31] D. C. Wharton, A. Tzagoloff, "Cytochrome oxidase from beef heart mitochondria," Methods in Enzymology, 10: 245-250 (1967).

[32] E. D. Chan, M. M. Chan, M. M. Chan, "Pulse oximetry: Understanding its basic principles facilitates appreciation of its limitations," Respiratory Medicine, 107(6): 789-799 (2013).

[33] R. Nosrati, S. Lin, V. Toronov, "Monitoring cerebral oxygenation and metabolism during cardiac arrest and CPR using hyperspectral near infrared spectroscopy (hNIRS),” Biomedical Optics 2016, OSA, paper JW3A.31 (2016).

[34] W. F. Cheong, S. A. Prahl, A. J. Welch, "A Review of the Optical Properties of Biological Tissue,” IEEE J Quantum Electronics, 26(12): 2166-2185 (1990).

[35] D. A. Boas, A. M. Dale, M. A. Franceschini, "Diffuse optical imaging of brain activation: approaches to optimizing image sensitivity, resolution and accuracy," NeuroImage, S275S288 (2004).

[36] V. Ntzaichristos, "Going deeper than microscopy: the optical imaging frontier in biology," Nature Methods, 7: 603-614 (2010).

[37] “Imagent,” ISS. Accessed: 2016-09-08, url: http://www.iss.com/biomedical/instruments/imagent.html.

[38] R. Nosrati, K. Vesely, T. A. Schweizer, V. Toronov, "Event-related changes of the prefrontal cortex oxygen delivery and metabolism during driving measured by hyperspectral fNIRS," Biomed Opt Express, 7(4): 1323-1335 (2016). 
[39] R. C. Haskell, L. O. Svaasand, T. Tsay, T. Feng, M. S. McAdams, B. J. Tromberg, "Boundary conditions for the diffusion equation in radiative transfer," JOSA A, 11(10): 2727-2741 (1994).

[40] M. Teplan, "Fundamentals of EEG Measurement," Measurement Science Review, 2(2) (2002).

[41] G. J. Tortora, B. H. Derrickson, “Principles of Anatomy and Physiology 14e,” Wiley (2014).

[42] N. Spruston, "Pyramidal neurons: dendritic structure and synaptic integration," Nature Reviews Neuroscience, 9: 206-221 (2008).

[43] D. W. Klass, “The Continuing Challenge of Artifacts in the EEG,” Am J EEG Technol,” 35: 239-269 (1995).

[44] N. Herrmann, "What is the function of the various brainwaves?" Scientific American (1997). Access: 2016-09-08, url: http://www.scientificamerican.com/article/what-is-thefunction-of-t-1997-12-22/.

[45] R. Widenhorn, M. M. Blouke, A. Weber, A. Rest, E. Bodegomá, “Temperature dependence of dark current in a CCD,” Proc. SPIE, 4669: 193-201 (2002).

[46] U. Voss, R. Holzmann, A. Hobson, W. Paulus, J. Koppehele-Gossel, A. Klimke, M. A. Nitsche, "Induction of self awareness in dreams through frontal low current stimulation of gamma activity," Nature Neuroscience, 17: 810-812 (2014).

[47] Y. Shao, G. Gu, Z. Jiang, W. Zhou, "Effects of Polynomial Trends on Detrending Moving Average Analysis," Fractals, 23(3) (2015). 
[48] A. Hyvarinen, E. Oja, "Independent Component Analysis: Algorithms and Applications," Neural Networks, 13(405): 411-430 (2000).

[49] K. Baek, B. A. Draper, J. R. Beverodge. K. She, "PCA vs. ICA: a comparison on the FERET data set," Proc. of the $4^{\text {th }}$ International Conference on Computer Vision, 824-827 (2002),

[50] B. Babusiak, J. Mohylova, "Eye-blink artifact detection in the EEG," World Congress on Medical Physics and Biomedical Engineering, 24: 1166-1169 (2009).

[51] V. Y. Toronov, X. Zhang, A. G. Webb, "Near-infrared study of the underlying physiology of the functional magnetic resonance signal in humans during hypoxia," Proc SPIE Int Soc Opt Eng, 5686: 543-546 (2005).

[52] P. Hartikainen, H. Soininen, J. Partanen, E. L. Helkala, P. Riekkinen, “Aging and spectral analysis of EEG in normal subjects: a link to memory and CSF AChE," Acta Neurol Scand, 86(2): 148-155 (1992).

[53] M. Maher, N. W. Churchill, A. L. de Oliveira Manoel, S. J. Graham, R. L. Macdonald, T. A. Schweizer, "Altered Resting-State Connectivity within Executive Networks after Aneurysmal Subarachnoid Hemorrhage," PLoS One, e0130483 (2015).

[54] D. Zhang, B. Liang, X. Wu, Z. Wang, P. Xu, S. Chang, B. Liu, M. Liu, R. Huang, "Directionality of large-scale resting-state brain networks during eyes open and eyes closed conditions," Front Hum Neruosci, 9:81 (2015).

[55] R. I. Goldman, J. M. Stern, J. E. Engel, M. S. Cohen, "Simultaneous EEG and fMRI of the alpha rhythm," Neuroreport, 13(18): 2487-2492 (2002). 
[56] E. Marx, A. Deutschlander, T. Stephan, M. Dieterich, M. Wiesmann, T. Brandt, "Eyes open and eyes closed as rest conditions: impact on brain actication patterns," NeuroImage, 21\{4\}: 1818-1824 (2004).

[57] K. K. Zakzanis, R. Mraz, S. J. Graham, “An fMRI study of the Trail Making Test,” Neurophyschologia, 43: 1878-1886 (2005).

[58] J. Moll, R. de Oliveira-Souza, F. T. Moll, I. E. Bramati, P. A. Andreiuolo, "The cerebral correlates of the set-shifting: An fMRI study of the trail making test," Arq NeuroPsiquiatr., 60(4): 900-905 (2002).

[59] K. Kirschfeld, "The physical basis of alpha waves in the electroencephalogram and the origin of the "Berger effect," Biol. Cybern, 92: 177-185 (2005).

[60] J. E. Resch, C. N. Brown, J. Schmidt, S. N. Macchiocchi, D. Blueitt, C. M. Cullum, M. S. Ferrara. "The sensitivity and specificity of clinical measures of sport concussion: three tests are better than one,” BJM Open Sport Exerc Med., 2: e000012 (2016).

[61] J. Song, C. Davey, C. Poulson, P. Luu, S. Turovets, E. Anderson, K. Li, D. Tucker. “EEG source localization: Sensor density and head surface coverage," Clinical Neuroscience, 256: 9-21 (2015).

[62] G. Buzsaki, A. Draguhn. "Neuronal Oscillations in Cortical Networks," Science, 304, 1926 (2004).

[63] B. Agrell, O. Dehlin. “The clock-drawing test,” Age and Aging, 27: 399-403 (1998).

[64] B. Uttl, C. Pilkenton-Taylor. "Letter cancellation performance across the adult life span," Clin Neuropsychol, 15(4): 521-530 (2001). 
[65] T.A. Salthouse. "What cognitive abilities are involved in trail-making performance?" Intelligence, 39(4): 222-232 (2011).

[66] F.A Fishburn, M.E. Norr, A.V. Medvedev, C.J. Vaidya. "Sensitivitty of fNIRS to cognitive state and load," Front Hum Neurosci, 8:76 (2014). 